\title{
Vascular flora of Serra do Japi Biological Reserve, Jundiaí, southeastern Brazil
}

\author{
Flora vascular da Reserva Biológica Municipal da Serra do Japi, Jundiaí, SP
}

\author{
Julio Antonio Lombardi ${ }^{1,2,5}$, Carolina da Silva Carvalho ${ }^{3}$, Leonardo Biral ${ }^{4}$, Mariana Naomi Saka ${ }^{4}$ \\ \& Sean Miki Hieda ${ }^{3}$
}

\begin{abstract}
Floristic sampling was carried out in Serra do Japi Biological Reserve, Jundiaí, state of São Paulo, Brazil, including physiognomies of semideciduous montane forest, a type of forest formation within the Atlantic Forest domain. Six hundred and sixty one species distributed in 129 families were recorded; the ten most diverse phanerogam families were: Asteraceae (56 species), Fabaceae (37), Rubiaceae (29), Poaceae (25), Solanaceae (24), Cyperaceae (19), Euphorbiaceae (19), Orchidaceae (19), Melastomataceae (17), and Piperaceae (17). The addition of 322 species from other floristic surveys increased the number of species of Serra do Japi to 976, distributed in 141 families. Compared to other floristic surveys carried out in the Atlantic Forest, our results highlight the floristic diversity of the study area and the importance of preserving the vegetation of this highly urbanized region.

Keywords: Brazil, semideciduous montane forest, floristic, Atlantic Forest, vascular plants.

Resumo

Amostragem florística foi feita em áreas da Reserva Biológica Municipal da Serra do Japi, Jundiaí, Estado de São Paulo, incluíndo fisionomias de floresta semidecídua montana, classificada como parte das formações da Mata Atlântica. Foram encontradas 661 espécies em 129 famílias, as dez fanerogâmicas mais diversas foram: Asteraceae (56 espécies), Fabaceae (37), Rubiaceae (29), Poaceae (25), Solanaceae (24), Cyperaceae (19), Euphorbiaceae (19), Orchidaceae (19), Melastomataceae (17), e Piperaceae (17). O acréscimo de 322 espécies adicionais compiladas de listagens florísticas suplementares eleva, para a Serra do Japi em geral, o número registrado a 976 espécies em 141 famílias. Comparada a outros levantamentos florísticos na Mata Atlântica, esses resultados demonstram a diversidade florística da área estudada e ressaltam o valor da preservação da vegetação nessa região altamente urbanizada.

Palavras-chave: Brasil, floresta semidecídua montana, florística, Mata Atlântica, plantas vasculares.
\end{abstract}

\section{Introduction}

The Atlantic Forest is highly complex, both floristically and structurally. In the past, it covered an almost continuous range along the coast, from the state of Rio Grande do Norte to the state of Rio Grande do Sul, which represents ca. $12 \%$ of the Brazilian territory (Consórcio Mata
Atlântica 1992; Fundação SOS Mata Atlântica \& INPE 2002; Galindo-Leal \& Câmara 2003). It comprises different vegetation types, from forests to fields. Its current legal circumscription is given by Decree no. 750/93, which deals with the use of the Atlantic Forest, defining it as forest formations and associated ecosystems inserted

\footnotetext{
This paper has additional data published in its electronic version.

${ }^{1}$ Universidade Estadual Paulista - UNESP, Instituto de Biociências de Rio Claro, Depto. Botânica, Av. 24-A 1515, 13506-900, Rio Claro, SP, Brazil.

${ }^{2}$ Research Productivity Fellowship CNPq, proccess 300240/2009-0.

${ }^{3}$ PIBIC Scholarship CNPq/UNESP, Undergraduate Program in Biological Sciences, Instituto de Biociências de Rio Claro, UNESP - Universidade Estadual Paulista, Av. 24-A 1515, 13506-900, Rio Claro, São Paulo, Brazil.

${ }^{4}$ CNPq Scholarship. Graduate Program in Biological Sciences (Plant Biology), Instituto de Biociências de Rio Claro, UNESP - Universidade Estadual Paulista, Av. 24-A 1515, 13506-900, Rio Claro, São Paulo, Brazil.

${ }^{5}$ Correponding author: e-mail: cissus@rc.unesp.br
} 
in the domain of the Atlantic Forest, with their respective official delimitations established (IBGE 1991).

The flora of the Atlantic Forest was estimated in 20 thousand species of vascular plants, of which approximately eight thousand would be endemic (Myers et al. 2000). Although this estimate allows us to place this formation within the so-called biodiversity hotspots (Mittermeier et al. 2004), we can still consider it relatively poorly known, in particular because from 1990 to 2006 more than 1,100 new species from this biome were described; over $40 \%$ of more than 1,100 new species described from Brazil were made from this biome (Sobral \& Stehmann 2009). Floristic inventories including non-tree species are relatively rare in the Atlantic Forest, whereas tree species are better known, mainly due to several phytosociological studies carried out in the state of São Paulo (e.g. Leitão-Filho 1982).

The Serra do Japi range is considered a priority area for conservation, representing the largest inland continuous forest of the state of São Paulo, and, despite the impacts of mining, logging and human occupation that it has undergone, it still represents an important remnant of the flora and fauna of southeastern Brazil (Joly 1992; LeitãoFilho \& Morellato 1997, Morellato 1992a).

The range is located in a highly populated region within the urban areas of São Paulo, Jundiaí, and Campinas, which potentializes the risks of disordered occupation that the area is subjected to. However, since 1983, Serra do Japi is under governmental trust (CONDEPHAAT - Conselho de Defesa do Patrimônio Histórico, Arqueológico, Artístico e Turístico do Estado/Council for the Defense of the Historical, Archaeological, Artistic and Touristic Heritage) and, from 1984 on, it was declared a reserve (APA - Área de Proteção Ambiental/Environmental Protection Area), partially located within the municipalities of Jundiaí and Cabreúva. The Serra do Japi Biological Reserve (RBMSJ - Reserva Biológica Municipal da Serra do Japi) was created in 1992 (Jundiaí 1991-1992), and in 1993 it was included in the Atlantic Forest Biosphere Reserve (LeitãoFilho \& Morellato 1997).

The Serra do Japi Biological Reserve has been extensively studied in terms of arboreal flora (Cardoso-Leite et al. 2002; Leitão-Filho 1992; Rodrigues et al. 1989), physiognomy (Cardoso-
Leite et al. 2005; Rodrigues \& Shepherd 1992), phenology (Morellato et al. 1989; Morellato et al. 1990; Morellato 1992b), and fauna (e.g. Brown Jr. 1992; Haddad \& Sazima 1992; Marinho-Filho 1992; Sazima \& Haddad 1992; Silva 1992).

The objective of the present study was to contribute to the knowledge of the flora of Serra do Japi Biological Reserve, creating a floristic list that includes all life forms, thus complementing the lists already published for the area and comparing it to other lists and taxonomic studies, aiming to check the presence of invasive, rare or endemic species, in order to give support to future conservation and management projects.

\section{Material and Methods}

Study area

The massif of Serra do Japi is located within the municipalities of Jundiaí, Itupeva, Cabreúva, Pirapora do Bom Jesus, and Cajamar, approximately between $23^{\circ} 11^{\prime} \mathrm{S}$ and $46^{\circ} 52^{\prime} \mathrm{W}$, and covers $354 \mathrm{~km}^{2}$ (Leitão-Filho \& Morellato 1997). The altitude of this mountain varies from 700 to $1,300 \mathrm{~m}$, and its average annual temperature varies from $15.7^{\circ} \mathrm{C}$ in the highest areas to $19.2^{\circ} \mathrm{C}$ in the lowest areas. July is the coldest month, with average temperatures between $11.8{ }^{\circ} \mathrm{C}$ and $15.3^{\circ} \mathrm{C}$; the warmest month is January, with average temperatures between $18.4^{\circ} \mathrm{C}$ and $22.2^{\circ} \mathrm{C}$. Hence, the local summer is warm and humid and the winter is cold and dry (Pinto 1992).

Serra do Japi Biological Reserve is located within the municipality of Jundiaí (between $23^{\circ} 12^{\prime}-23^{\circ} 21^{\prime} \mathrm{S}$ and $46^{\circ} 30^{\prime}-46^{\circ} 05^{\prime} \mathrm{W}$ ), and comprises $2,071.20$ ha (Fig. 1) covered mainly by seasonal semideciduous montane forest (CardosoLeite et al. 2005; IBGE 1991; Jundiaí 1991-1992).

Collections of botanical material were carried out monthly, from May 2007 to November 2008; only reproductive specimens were collected. Marked trails were covered, with eventual incursions into the surrounding vegetation. Considering that previous inventories focused on sampling the tree stratum, we focused on sampling specimens of other life forms, mainly herbs and lianas.

The samples were pressed and dried following Fidalgo \& Bononi (1984) and notes on life form, height, color, and other characters were taken. Exsiccates were deposited in the collection of Herbarium Rioclarense (HRCB), 


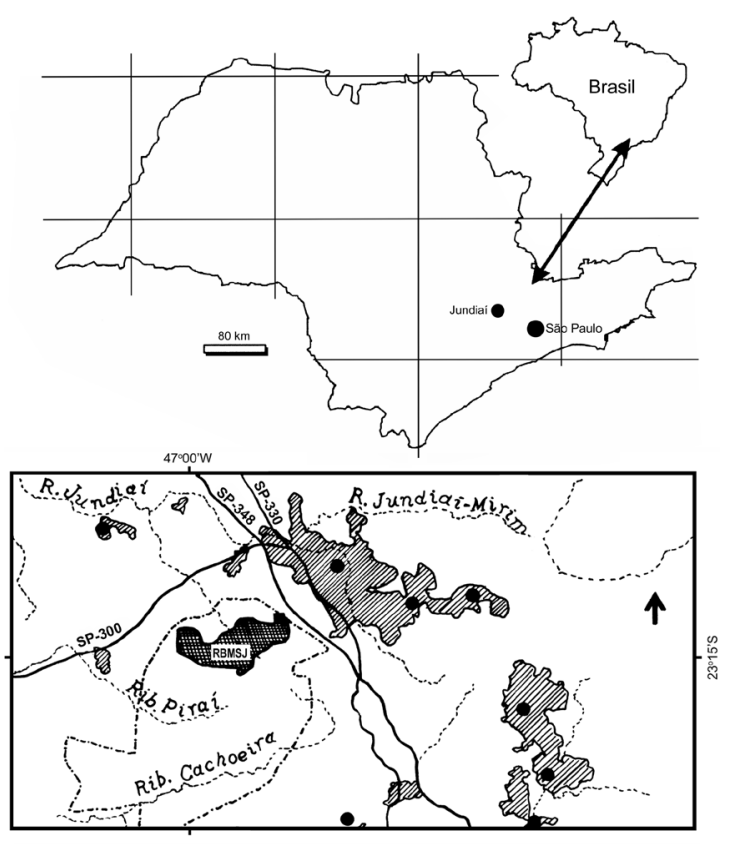

Figura 1 - Location of the municipality of Jundiaí in the state of São Paulo and of Serra do Japi Biological Reserve, showing the surrounding urban areas (adapted from Cardoso-Leite et al. 2005 and Neves \& Cardoso 2006).

of the Institute of Bioscience of Rio Claro, Universidade Estadual Paulista (UNESP). Species were identified based on the literature, exchange of duplicates and consultation with specialists, as well as by comparison with identified specimens deposited in other herbaria in the state of São Paulo (Appendix 1). The abbreviations of herbaria followed Thiers (2009). The classification of angiosperms followed APG III (2009), whereas the classification of Lycophyta, Monilophyta, and gymnosperms followed, respectively, Kramer \& Tryon (1990), Smith et al. (2006), and Page (1990).

The floristic list presented encompasses species collected in this inventory, plus species reported as occurring in the area in previously published lists for Serra do Japi (Cardoso-Leite et al. 2002; Leitão-Filho 1992; Pansarin \& Pansarin 2008; Rodrigues et al. 1989). Vouchers are mentioned only for collections carried out in the present study.

Names of native species included in the lists presented here were checked on the Lista de
Espécies da Flora do Brasil (Forzza et al. 2010). The botanical synonymy and authorship of species were checked in the literature (Forzza et al. 2010, "The Plant List"), and through consultation with specialists. Whenever necessary, updating and correction were made.

In the floristic comparison among Atlantic Forest areas (Braga 2005), only species collected in the present inventory were considered; the six other areas of Atlantic Forest s.l. used for comparison were selected from papers published in the past ten years, which were extensive and not restricted to a particular life form: Araldo Forest (Mata do Araldo), state of Paraná (Souza \& Monteiro 2005); Santa Genebra Municipal Reserve (Reserva Municipal de Santa Genebra), Campinas, state of São Paulo (Guaratini et al. 2008); Caratinga Biological Station (Estação Biológica de Caratinga) and Rio Doce State Park (Parque Estadual do Rio Doce), state of Minas Gerais (Lombardi \& Gonçalves 2000); João Vasconcelos-Sobrinho Ecological Park (Parque Ecológico João Vasconcelos-Sobrinho), state of Pernambuco (Rodal \& Sales 2007); Intervales State Park (Parque Estadual Intervales), Saibadela Base, state of São Paulo (Zipparro et al. 2005); Toró Forest (Mata do Toró), state of Permanbuco (Rodal et al. 2005).

\section{Results and Discussion}

In the Serra do Japi Biological Reserve, 661 species from 129 families (Appendix 1) were collected. The ten best-represented phanerogam families were: Asteraceae (56 species), Fabaceae (37), Rubiaceae (29), Poaceae (25), Solanaceae (24), Cyperaceae (19), Euphorbiaceae (19), Orchidaceae (19), Melastomataceae (17), and Piperaceae (17). Among pteridophytes, the three most important families were: Polypodiaceae, Pteridaceae (11 species each), and Blechnaceae (6).

The addition of 322 species compiled from other floristic lists increased the number of species recorded for Serra do Japi to 976 species from 141 families. Compared to the other inventories selected (Tab. 1), the number of species sampled in the present study was only smaller than that reported in the inventory of the Atlantic Forest of Minas Gerais (Lombardi \& Gonçalves 2000), which includes two areas: Caratinga Biological 
Station (Estação Biológica de Caratinga) and Rio Doce State Park (Parque Estadual do Rio Doce). The ten most common families were also present in the other inventories used for comparison; Fabaceae was the most important family in terms of number of species in eight inventories, whereas in Serra do Japi Biological Reserve the predominance of Asteraceae over Fabaceae is explained by the inclusion of ruderal species in the present study.

Although all sampled areas exhibited different signs of anthropic alteration, the large number of species found in this study highlights the high floristic richness of the region. Additional collections could add more species to the list presented here.

The identification of the specimens sampled in Serra do Japi Biological Reserve recorded species previously considered extinct in the state: Prestonia solanifolia (Müll.Arg.) Woodson and Peperomia nitida Dahlst. (Mamede et al. 2007), as well as several species considered vulnerable: Cissampelos pareira L., Dicksonia sellowiana Hook., Eugenia brasiliensis Lam., Euterpe edulis Mart., and Trichilia hirta L. (Mamede et al. 2007).

Cissus striata subsp. argentina (Suess.) Lombardi was observed in the region, which had been previously recorded for the state of São Paulo only in collections from the municipalities of São Bernardo do Campo and Campos do Jordão, where it was found up to the Caparaó Mountain Range (Serra do Caparaó), state of Minas Gerais, always in high-altitude areas, but with its geographic distribution restricted mainly to southern Brazil and northern Argentina (Lombardi 2000, 2002). Moreover, a new species, Sida sp. nov., was discovered and is being described (A. Krapovickas, personal communication).

In Serra do Japi Biological Reserve, 112 of the collected species can be classified as cultivated, both non-invasive (29 species) or invasive ( 83 species) (Appendix 1).

The species considered as cultivated noninvasive were collected in the surroundings of the Ecological Base (head office) or in ruins of abandoned residences, and were apparently unable to self-propagate, since other individuals outside these sites have not been observed. These species, according to Colautti \& MacIsaac
(2004), are non-invasive and classified in stages III and IVb, non-native species that, respectively, have restricted populations and are numerically rare, or are locally restricted but dominant in the area.

The species considered as invasive here are non-native with a capacity for self-propagation, represented by widely distributed individuals and apparently maintaining a population in the study areas, which, according to Colautti \& MacIsaac (2004), are invasive species classified in stages IVa and $\mathrm{V}$, non-native species that are well distributed in the study area and, respectively, rare or dominant. The vast majority of these species are herbs, few are ornamental woody or fruitbearing. Especially common along the trails and most illuminated areas are the herbs Impatiens walleriana Hook.f., very common along trails and borders, particularly in humid areas, and Crocosmia crocosmiiflora (Lemoine ex Morren) N.E.Br. that occurs in more restricted areas, mainly along the trail Ecological Base-Belvedere (Base Ecológica-Mirante), which has more intense visitation. Some tree or shrub species, such as Eriobotrya japonica (Thunb.) Lindl. and Citrus limon (L.) Burm.f., are sometimes found growing in the forest; the dispersal of these species is probably carried out by the autochthonous fauna, such as birds, taking into account that they are dispersed in the region.

We considered these invasive species to be integrated in the ecosystem, in a 'natural' evolution under human interference; according to some authors, efforts to amend situations such as these are useless and considered a waste of resources (Hobbs et al. 2006). Eradicating these species in order to restore the ecosystem to a previous condition is probably very difficult, or even impossible, since these species are well distributed in the study areas, though they occupy areas of relatively narrow ecological range, on borders and humid areas, where they can be rare (stage IVa: Eriobotrya japonica and Citrus limon) or dominant (stage V: Impatiens walleriana and Crocosmia crocosmiiflora).

The species richness observed in the Serra do Japi Biological Reserve shows the great importance of this area for biodiversity conservation in a region of the state of São Paulo where few areas with extensive native vegetation are found. The conservation of this important 
照造言客

का चै

ชึ

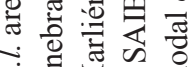

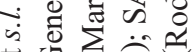

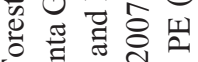

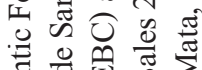

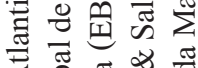

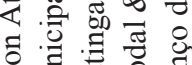

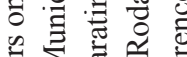

ए $\sum_{0}$

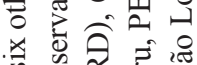

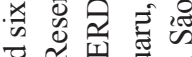

일

药

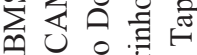

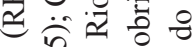

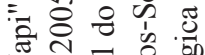

जे

윤

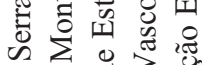

तु $\nsim$ है

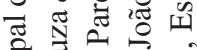

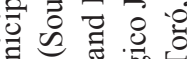

番

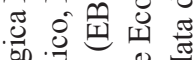

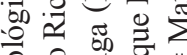

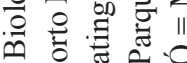

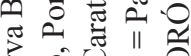

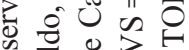

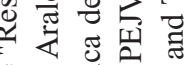

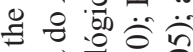

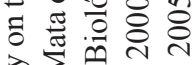

उ) $\sum_{0}$ त

娄

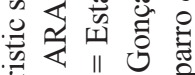

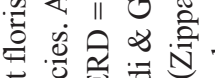

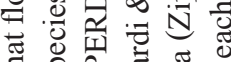

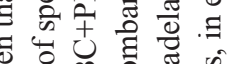

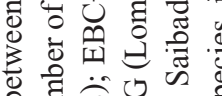

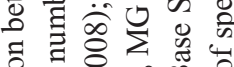

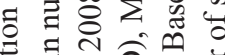

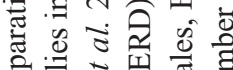

응 을

记要目造焉

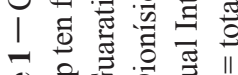

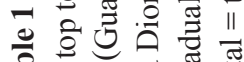

过荡

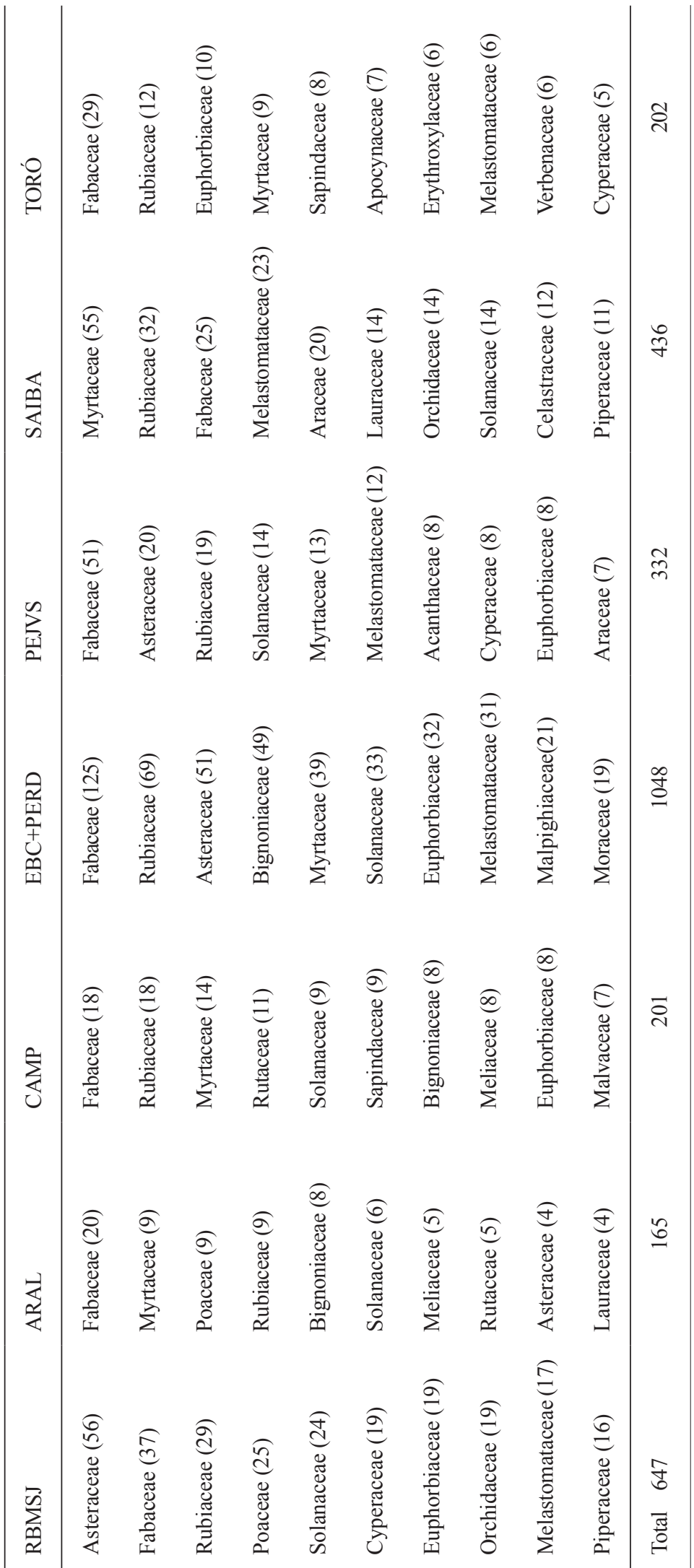


reserve with 2,071.20 ha of vegetation, despite the alterations that it has undergone so far, is even more urgent in a region where urbanization and industrialization are very intensive (Florgård 2004). Furthermore, it is very important to preserve headwaters and to promote well-being in the region (Gowdy 1997; Kellert 1993; Pretty 2004; Ulrich 1993).

\section{Acknowledgments}

FAPESP - São Paulo Research Foundation (process no. 2006/07225-4) funded this research. CNPq - National Council for Scientific and Technological Development granted JAL (process number 306395/2006-1) a research productivity fellowship and CSC, MNS, and SMH undergraduate research scholarships. Jundiaí City Administration (Prefeitura Municipal de Jundiaí) and the Municipal Department of Planning and Environment (Secretaria Municipal de Planejamento e Meio Ambiente) granted us a permit for carrying out the present study. We also thank one anonymous reviewer, whose criticisms improved substantially the writing style of the manuscript. João Vasconcellos Neto helped us elaborate the original project. Natália Macedo Ivanauskas gave us invaluable suggestions for an early version of the manuscript. The specialists reported in the species list helped identifying species.

\section{References}

APG - Angiosperm Phylogeny Group. 2009. An update of the Angiosperm Phylogeny Group classification for the orders and families of flowering plants: APG III. Botanical Journal of the Linnean Society of London 161: 105-121.

Braga, J.M.A. 2005. Marantaceae - novidades taxonômicas e nomenclaturais III: tipificações, sinonímias e uma nova combinação em Calathea. Acta Botanica Brasilica 19: 763-768.

Brown Jr., K.S. 1992. Borboletas da Serra do Japi: diversidade, hábitos, recursos alimentares e variação temporal. In: Morellato, L.P.C. (ed.) História natural da Serra do Japi. Editora da UNICAMP, FAPESP, Campinas. Pp. 142-186.

Cardoso-Leite, E.; Pagani, M.I.; Hamburger, D.S. \& Monteiro, R.R. 2002. Fitofisionomia, fitossociologia e conservação da vegetação na Reserva Biológica Municipal da Serra do Japi, Jundiaí, SP. Naturalia (Rio Claro) 27: 165-200.

Cardoso-Leite, E.; Pagani, M.I.; Monteiro, R.R. \& Hamburger, D.S. 2005. Ecologia da paisagem: mapeamento da vegetação da Reserva Biológica da Serra do Japi, Jundiaí, SP, Brasil. Acta Botanica Brasílica 19: 233-243.

Consórcio Mata Atlântica. 1992. Reserva da Biosfera da Floresta Atlântica. Plano de ação. Referências básicas. Vol. 1. Editora da Universidade Estadual de Campinas, Campinas. 147p.

Colautti, R.I. \& MacIsaac, H.J. 2004. A neutral terminology to define "invasive" species. Diversity and Distributions 10: 135-141.

Forzza, R. C. et al. 2010. Lista de Espécies da Flora do Brasil. 2010 [continuamente atualizado]. Available in $<$ http://floradobrasil.jbrj.gov.br/2010/>. Access on 16 May 2011.

Fidalgo, O. \& Bononi, V.L.R. 1984. Técnicas de coleta, preservação e herborização de material botânico. Instituto de Botânica, São Paulo. 62p.

Florgård, C. 2004. Remaining original natural vegetation in towns and cities - Introduction. Urban Forestry \& Urban Greening 3: 1-2.

Fundação SOS Mata Atlântica \& INPE. 2002. Atlas dos remanescentes florestais da Mata Atlântica; período 1995-2000. Available in <http://mtc-m12.sid.inpe. br/col/sid.inpe.br/jeferson/2003/06.02.07.45/doc/ RelatorioAtlas.pdf $>$. Access on 20 Jun 2012.

Galindo-Leal, C. \& Câmara, I.G. 2003. Atlantic Forest hotspots status: an overview. In: Galindo-Leal, C. \& Câmara, I.G. (eds.). The Atlantic Forest of South America: biodiversity status, threats, and outlook. Center for Applied Biodiversity Science e Island Press, Washington. Pp. 3-11.

Gowdy, J. M. 1997. The value of biodiversity: markets, society, and ecosystems. Land Economics 73: 25-41.

Guaratini, M.T.G.; Gomes, E.P.C.; Tamashiro, J.Y. \& Rodrigues, R.R. 2008. Composição florística da Reserva Municipal de Santa Genebra, Campinas, SP. Revista Brasileira de Botânica 31: 323-337.

Haddad, C.F.B. \& Sazima, I. 1992. Anfíbios anuros da Serra do Japi. In: Morellato, L.P.C. (ed.). História Natural da Serra do Japi. Editora da UNICAMP, FAPESP, Campinas. Pp. 188-211.

Hobbs, R.J.; Arico, S.; Aronson, J.; Baron, J.S.; Bridgewater, P.; Cramer, V.A.; Epstein, P.R.; Ewel, J.J.; Klink, C.A.; Lugo, A.E.; Norton, D.; Ojima, D.; Richardson, D.M.; Sanderson, E.W.; Valladares, F.; Vilà, M.; Zamora, R. \& Zobel, M. 2006. Novel ecosystems: theoretical and management aspects of the new ecological world order. Global Ecology \& Biogeography 15: 1-7.

IBGE - Fundação Instituto Brasileiro de Geografia e Estatística. 1991. Manual técnico da vegetação brasileira. Série Manuais Técnicos em Geociências. Secretaria do Orçamento e Coordenação da Presidência da República, Rio de Janeiro. 92p. 
Joly, C.A. 1992. A preservação da Serra do Japi. In: Morellato, L.P.C. (ed.). História natural da Serra do Japi. Editora da UNICAMP, FAPESP, Campinas. Pp. 310-321.

Jundiaí (Município). Lei no 3.672, de 10 de janeiro de 1991. Cria a Reserva Biológica Municipal da Serra do Japi. Decreto n ${ }^{\circ} 13.196$, de 30 de dezembro de 1992. Available in <http://www.jundiai.sp.gov. br/PMJSITE/biblio.nsf/V03.01/lei_3672-91/\$file/ lei_3672-91.pdf $>$. Access on $28 \mathrm{Jul} 2009$.

Kellert, S.R. 1993. The biological basis for human values of nature. In: Kellert, S.R. \& Wilson, E.O. (eds.). The biophilia hypothesis. Island Press, Washington. Pp. 42-69.

Kramer, K.U. \& Tryon, R.M. 1990. Introduction to the treatment of pterodophytes. In: Kramer, K.U. \& Green, P.S. (eds.). The families and genera of vascular plants. I. Pteridophytes and Gymnosperms. Springer-Verlag, Berlin. Pp. 12-13.

Leitão-Filho, H.F. 1982. Aspectos taxonômicos das florestas do estado de São Paulo. Silvicultura em São Paulo 16: 197-206.

Leitão-Filho, H.F. 1992. A flora arbórea da Serra do Japi. In: Morellato, L.P.C. (ed.). História natural da Serra do Japi. Editora da UNICAMP, FAPESP, Campinas. Pp. 40-62.

Leitão-Filho, H.F. \& Morellato, L.P.C. 1997. Semideciduous forests of Southeastern Brasil Serra do Japi. In: Davis, S.D.; Heywood, V.H.; Herrera-MacBryde, O.; Villa-Lobos, J. \& Hamilton, A.C. (eds.). Centres for plant diversity: a guide and strategy for their conservation. IUCN, WWF, Washington. Pp. 381-384.

Lombardi, J.A. 2000. Vitaceae-gêneros Ampelocissus, Ampelopsis e Cissus. Flora Neotropica Monograph 80: 1-250.

Lombardi, J.A. 2002. Vitaceae. In: Wanderley, M.G.L.; Shepherd, G.J.; Giulietti, A.M.; Melhem, T.S.; Bittrich, V. \& Kameyama, C. Flora fanerogâmica do estado de São Paulo. HUCITEC, FAPESP, São Paulo. Vol. 2. Pp. 365-374.

Lombardi, J.A \& Gonçalves, M. 2000. Composição florística de dois remanescentes de Mata Atlântica do sudeste de Minas Gerais, Brasil. Revista Brasileira de Botânica 23: 255-282.

Mamede, M.C.H.; Souza, V.C.; Prado, J.; Barros, F.; Wanderley, M.G.L. \& Rando, J.G. 2007. Livro vermelho das espécies vegetais ameaçadas do Estado de São Paulo. Instituto de Botânica; Imprensa Oficial, São Paulo. 165p.

Marinho-Filho, J. 1992. Mamíferos da Serra do Japi. In: Morellato, L.P.C. (ed.). História natural da Serra do Japi. Editora da UNICAMP, FAPESP, Campinas. Pp. 264-286.

Mittermeier, R.A.; Gil, P.R.; Hoffmann, M.; Pilgrim, J.; Brooks, J.; Mittermeier, C.G.; Lamourux, J. \&
Fonsêca, G.A.B. 2004. Hotspots revisited: Earth's biologically richest and most endangered terrestrial ecoregions. CEMEX, Washington, D.C.

Morellato, L.P.C. 1992a. Introdução. In: Morellato, L.P.C. (ed.). História natural da Serra do Japi. Editora da UNICAMP, FAPESP, Campinas. Pp. 8-11.

Morellato, L.P.C. 1992b. Sazonalidade e dinâmica de ecossistemas florestais na Serra do Japi. In: Morellato, L.P.C. (ed.). História natural da Serra do Japi. Editora da UNICAMP, FAPESP, Campinas. Pp. 98-110.

Morellato, L.P.C.; Rodrigues, R.R. ; Leitão-Filho, H.F.; Joly, C.A. 1989. Estudo comparativo da fenologia de espécies arbóreas de floresta de altitude e Floresta Mesófila Semidecídua na Serra do Japi, SP. Revista Brasileira de Botânica 12: 85-98.

Morellato, L.P.C.; Rodrigues, R.R.; Leitão-Filho, H.F. \& Joly, C.A. 1990. Estratégias fenológicas de espécies arbóreas em floresta de altitude na Serra do Japi, Jundiaí, SP. Revista Brasileira de Biologia 50: 149-162.

Myers, N.; Mittermeier, R.A.; Mittermeier, C.G.; Fonsêca, G.A.B. \& Kent, J. 2000. Biodiversity hotspots for conservation priorities. Nature 403: 853-858.

Neves, M. A. \& Cardoso, M. S. N. 2006. Qualidade das águas do Rio Jundiaí (SP): a necessidade de uma gestão integrada. Holos Environment [Online] 6:2. Available in <http://cecemca.rc.unesp.br/ojs/ index.php/holos/article/view/556/467>. Access on 5 Oct 2009.

Page, C.N. 1990. Taxonomic concepts in Conifers and Ginkgoids. In: Kramer, K.U. \& Green, P.S. (ed.). The families and genera of vascular plants. I. Pteridophytes and Gymnosperms. Springer-Verlag, Berlin. Pp. 282.

Pansarin, E.R. \& Pansarin, L.M. 2008. A família Orchidaceae na Serra do Japi, São Paulo, Brasil. Rodriguésia 59: 99-111.

Pinto, H.S. 1992. Clima da Serra do Japi. In: Morellato, L.P.C. (ed.). História natural da Serra do Japi. Editora da UNICAMP, FAPESP, Campinas. Pp. 30-38.

Pretty, J. 2004. How nature contributes to mental and physical health. Spirituality and Health International 5: 68-78.

Rodal, M.J.N.; Lucena, M.F.A.; Andrade, K.V.S.A. \& Melo, A.L. 2005. Mata do Toró: uma floresta estacional semidecidual de terras baixas no nordeste do Brasil. Hoehnea 32: 283-294.

Rodal, M.J.N. \& Sales, M.F. 2007. Composição da flora vascular em um remanescente de floresta montana no semi-árido do nordeste do Brasil. Hoehnea 34: 433-446.

Rodrigues, R.R. \& Shepherd, G.J. 1992. Análise da variação estrutural e fisionômica da vegetação e 
características edáficas, num gradiente altitudinal na Serra do Japi. In: Morellato, L.P.C. (ed.). História Natural da Serra do Japi. Editora da UNICAMP \& FAPESP, Campinas. Pp. 64-96.

Rodrigues, R.R.; Morellato, L.P.C.; Joly, C.A. \& LeitãoFilho, H.F. 1989. Estudo florístico e fitossociológico envolvendo um gradiente altitudinal em uma mata mesófila estacional semidecídua na Serra do Japi, Jundiaí, SP. Revista Brasileira de Botânica 12: 71-84.

Sazima, I. \& Haddad, C.F.B. 1992. Répteis da Serra do Japi: notas sobre história natural. In: Morellato, L.P.C. (ed.). História natural da Serra do Japi. Editora da UNICAMP, FAPESP, Campinas. Pp. 212-236.

Silva, W.R. 1992. As aves da Serra do Japi. In: Morellato, L.P.C. (ed.). História natural da Serra do Japi. Editora da UNICAMP, FAPESP, Campinas. Pp. 238-263.

Smith, A.R.; Pryer, K.M.; Schuettpelz, E.; Korall, P.; Schneider, H. \& Wolf, P.G. 2006. A classification for extant ferns. Taxon 55: 705-731.

Sobral, M. \& Stehmann, J.R. 2009. An analysis of new angiosperm species discoveries in Brazil (1990-2006). Taxon 58: 227-232.
Souza, M.C. \& Monteiro, R. 2005. Levantamento florístico em remanescente de floresta ripária no alto rio Paraná: Mata do Araldo, Porto Rico, Paraná, Brasil. Acta Scientiarum Biological Sciences 27: 405-414.

The Plant List. 2010. Version 1. Available in <http:// www.theplantlist.org/>. Access on 27 May 2011.

Thiers, B. 2009 "continuously updated". Index Herbariorum: A global directory of public herbaria and associated staff. New York Botanical Garden's Virtual Herbarium. Available in $<$ http:// sweetgum.nybg.org/ih/>. Access on 22 Jul 2009.

Ulrich, R. 1993. Biophilia, biophobia, and natural landscapes. In: Kellert, S.R. \& Wilson, E.O. (ed.). The biophilia hypothesis. Island Press, Washington. Pp. 73-137.

Zipparro, V.B.; Guilherme, F.A.G.; Almeida-Scabbia, R.J. \& Morellato, L.P.C. 2005. Levantamento florístico de Floresta Atlântica no sul do estado de São Paulo, Parque Estadual Intervales, Base Saibadela. Biota Neotropica 5: 147-170. Available in $<$ http://www.biotaneotropica.org.br/v5n1/pt/abs tract?inventory+BN02605012005>. Access on 24 Aug 2010. 


\section{Vascular flora of Serra do Japi Biological Reserve, Jundiaí, southeastern Brazil}

Flora vascular da Reserva Biológica Municipal da Serra do Japi, Jundiaí, SP

Julio Antonio Lombardi ${ }^{1,2,5}$, Carolina da Silva Carvalho ${ }^{3}$, Leonardo Biral ${ }^{4}$, Mariana Naomi Saka ${ }^{4}$

\& Sean Miki Hieda ${ }^{3}$

Appendix 1 - Floristic list from the "Reserva Biológica Municipal da Serra do Japi". JAL = collections of J.A. Lombardi et al.; LBS = collections of L.B. Santos. ${ }^{1,2,3,4}=$ species reported by Cardoso-Leite et al. $(2002)^{1}$, Leitão-Filho (1992) $)^{2}$, Rodrigues et al. $(1989)^{3}$ or Pansarin \& Pansarin $(2008)^{4}$, but not sampled in the present study. $\#=$ species only found in cultivation; $\S=$ invasive species.

\begin{tabular}{|c|c|c|}
\hline Família & Espécie & Voucher \\
\hline \multirow[t]{11}{*}{ Acanthaceae } & Aphelandra longiflora (Lindl.) Profice & JAL 7369 \\
\hline & A. schottiana (Nees) Profice & JAL 6757 \\
\hline & Eranthemum pulchellum Andrews\# & JAL 7437 \\
\hline & Hygrophila costata Nees & JAL 7309 \\
\hline & Justicia carnea Lindl. & JAL 6628 \\
\hline & J. lythroides (Nees) V.A.W.Graham & JAL 7304 \\
\hline & Justicia sp. 1 & JAL 6637 \\
\hline & Mendoncia velloziana Mart. & JAL 7006 \\
\hline & Pseuderanthemum sp. 1 & JAL 6751 \\
\hline & Ruellia sp. 1 & JAL 7418 \\
\hline & Indet. 1 & JAL 7333 \\
\hline Adoxaceae & Sambucus nigra L."\# & JAL 6845 \\
\hline Alliaceae & Nothoscordum gracile (Aiton) Stearn var. gracile & JAL 7519 \\
\hline \multirow[t]{2}{*}{ Alstroemeriaceae } & Alstroemeria cunha Vell. & JAL 7312 \\
\hline & Bomarea edulis (Tussac.) Herb. & JAL 7059 \\
\hline \multirow[t]{7}{*}{ Amaranthaceae } & Alternanthera tenella Colla & JAL 6737 \\
\hline & Chamissoa altissima (Jacq.) Kunth. var. altissima & JAL 6759 \\
\hline & Chenopodium ambrosioides $\mathrm{L} . \S$ & JAL 7299 \\
\hline & Hebanthe eriantha (Poir.) Pedersen & JAL 6856 \\
\hline & Iresine diffusa Humb. \& Bonpl. ex Willd. & JAL 7361 \\
\hline & Pfaffia gnaphaloides (L.f.) Mart. & JAL 6598 \\
\hline & Indet. 1 & JAL 6919 \\
\hline \multirow{2}{*}{$\begin{array}{l}\text { Amaryllidaceae (J.H.A. } \\
\text { Dutilh - UEC) }\end{array}$} & Hippeastrum puniceum (Lam.) Kuntze & JAL 6914 \\
\hline & Hippeastrum sp. [híbrido cultivado] ${ }^{*}$ & JAL 7520 \\
\hline \multirow[t]{5}{*}{ Anacardiaceae } & Astronium graveolens Jacq. ${ }^{2,3}$ & \\
\hline & Lithraea molleoides (Vell.) Engl..$^{1,2}$ & \\
\hline & Schinus terebinthifolius Raddi & JAL 6651 \\
\hline & Tapirira guianensis Aubl. & JAL 6898 \\
\hline & T. obtusa (Benth.) J.D. Mitch., ${ }^{2,3}$ & \\
\hline
\end{tabular}




\begin{tabular}{|c|c|c|}
\hline Família & Espécie & Voucher \\
\hline \multirow{2}{*}{$\begin{array}{l}\text { Anemiaceae (A. Salino - } \\
\text { BHCB) }\end{array}$} & Anemia phyllitidis (L.) Sw. & JAL 6647 \\
\hline & A. raddiana Link & JAL 7290 \\
\hline \multirow[t]{4}{*}{ Annonaceae } & Annona cacans Warm. ${ }^{2,3}$ & \\
\hline & A. sylvatica A.St.-Hil. & JAL 6974 \\
\hline & Guatteria australis A.St.-Hil. & JAL 7028 \\
\hline & Xylopia brasiliensis Spreng. ${ }^{2}$ & \\
\hline \multirow[t]{3}{*}{ Apiaceae } & Centella asiatica (L.) Urb. ${ }^{\S}$ & JAL 6953 \\
\hline & Cyclospermum leptophyllum (Pers.) Sprague $e^{\S}$ & JAL 6792 \\
\hline & Eryngium horridum Malme & JAL 6886 \\
\hline \multirow{16}{*}{$\begin{array}{l}\text { Apocynaceae (R. Morokawa, } \\
\text { W. Marcondes-Ferreira - } \\
\text { UEC) }\end{array}$} & Asclepias curassavica L.» & JAL 6795 \\
\hline & Aspidosperma olivaceum Müll.Arg. & JAL 7476 \\
\hline & A. parvifolium A.DC. ${ }^{1}$ & \\
\hline & A. pyricollum Müll.Arg. ${ }^{3}$ & \\
\hline & Condylocarpon isthmicum (Vell.) A.DC. & JAL 6458 \\
\hline & Ditassa burchellii Hook. \& Arn. & JAL 7314 \\
\hline & D. hispida (Vell.) Fontella & JAL 7313 \\
\hline & Ditassa sp. 1 & JAL 7425 \\
\hline & Odontadenia sp. 1 & JAL 7317 \\
\hline & Orthosia urceolata E.Fourn. & JAL 6823 \\
\hline & Oxypetalum insigne (Decne.) Malme var. insigne & JAL 6719 \\
\hline & O. pachyglossum Decne. & JAL 7074 \\
\hline & Peltastes peltatus (Vell.) Woodson & JAL 7024 \\
\hline & Prestonia coalita (Vell.) Woodson & JAL 7048 \\
\hline & P. solanifolia (Müll.Arg.) Woodson & JAL 6989 \\
\hline & Rauvolfia sellowii Müll.Arg. ${ }^{2}$ & \\
\hline \multirow[t]{2}{*}{ Aquifoliaceae } & Ilex cerasifolia Reissek & JAL 7291 \\
\hline & I. dumosa Reissek $^{1}$ & \\
\hline \multirow{11}{*}{$\begin{array}{l}\text { Araceae (L.G. Temponi- } \\
\text { UNIOESTE, M. Nadruz - RB) }\end{array}$} & Anthurium gaudichaudianum Kunth & JAL 6691 \\
\hline & A. scandens (Aubl.) Engl. & JAL 6612 \\
\hline & A. sellowianum Kunth & JAL 8646 \\
\hline & Asterostigma sp. 1 & JAL 7249 \\
\hline & Dieffenbachia amoena hort. ex Gentil ${ }^{*}$ & JAL 7349 \\
\hline & Monstera adansonii var. klotzschiana (Schott) Madison & JAL 6964 \\
\hline & Philodendron appendiculatum Nadruz \& Mayo & JAL 7487 \\
\hline & P. bipinnatifidum Schott & JAL 7451 \\
\hline & P. martianum Engl. ${ }^{\#}$ & JAL 6965 \\
\hline & Xanthosoma sp. 1 & JAL 7414 \\
\hline & Zantedeschia aethiopica (L.) Spreng." & JAL 7470 \\
\hline \multirow[t]{3}{*}{ Araliaceae } & Dendropanax cuneatus (DC.) Decne. \& Planch. & JAL 6758 \\
\hline & Hydrocotyle quinqueloba Ruiz \& Pav. & JAL 6980 \\
\hline & Schefflera calva (Cham.) Frodin \& Fiaschi ${ }^{1,2}$ & \\
\hline
\end{tabular}




\begin{tabular}{|c|c|c|}
\hline Família & Espécie & Voucher \\
\hline Araucariaceae & Araucaria angustifolia (Bertol.) Kuntze & JAL 7379 \\
\hline \multirow{4}{*}{ Arecaceae } & Acrocomia aculeata (Jacq.) Lodd. ex Mart. $^{2}$ & \\
\hline & Bactris setosa Mart. & JAL 6999 \\
\hline & Euterpe edulis Mart. $^{2}$ & \\
\hline & Syagrus romanzoffiana (Cham.) Glassman ${ }^{2}$ & \\
\hline Aristolochiaceae & Aristolochia triangularis Cham. \& Schltdl. & JAL 6613 \\
\hline Aspagaraceae & Yucca filamentosa L.\# & JAL 7505 \\
\hline \multirow{3}{*}{$\begin{array}{l}\text { Aspleniaceae (T.E. Almeida, } \\
\text { A. Salino-BHCB) }\end{array}$} & Asplenium claussenii Hieron. & JAL 6660 \\
\hline & A. mucronatum C.Presl & JAL 8649 \\
\hline & A. triquetrum N.Murak. \& R.C.Moran & JAL 6822 \\
\hline \multirow{31}{*}{$\begin{array}{l}\text { Asteraceae (M.E. Monge - } \\
\text { UEC) }\end{array}$} & Acanthospermum australe (Loefl.) Kuntze ${ }^{\S}$ & JAL 7337 \\
\hline & Achyrocline satureioides (Lam.) DC. & JAL 6806 \\
\hline & Ambrosia polystachya DC. ${ }^{\S}$ & JAL 6945 \\
\hline & Baccharis articulata (Lam.) Pers. & JAL 6877 \\
\hline & B. crispa Spreng. & JAL 6817 \\
\hline & B. dentata (Vell.) G.M. Barroso & JAL 7063 \\
\hline & B. dracunculifolia DC. & JAL 7274 \\
\hline & B. mesoneura DC. & JAL 6807 \\
\hline & $B$. aff. reticularia $\mathrm{DC}$. & JAL 7447 \\
\hline & B. semiserrata DC. & JAL 6821 \\
\hline & B. tarchonanthoides DC. & JAL 6895 \\
\hline & B. trinervis (Lam.) Pers. & JAL 6881 \\
\hline & Barrosoa aff. betonicaeformis (DC.) R.M.King \& H.Rob. & JAL 7464 \\
\hline & Calea pinnatifida (R.Br.) Less. & JAL 6470 \\
\hline & Campovassouria cruciata (Vell.) R.M.King \& H.Rob. & JAL 6859 \\
\hline & Chaptalia nutans (L.) Pol. ${ }^{\S}$ & JAL 6797 \\
\hline & Coreopsis lanceolata L. & JAL 6977 \\
\hline & Cosmos sulphureus Cav. ${ }^{\S}$ & JAL 6755 \\
\hline & Cyrtocymura scorpioides (Lam.) H. Rob. & JAL 6805 \\
\hline & Dasyphyllum brasiliense (Spreng.) Cabrera ${ }^{2}$ & \\
\hline & D. flagellare (Casar.) Cabrera & JAL 7443 \\
\hline & Dendrophorbium pellucidinerve (Sch.Bip. ex Baker) C.Jeffrey & JAL 6809 \\
\hline & Elephantopus mollis Kunth ${ }^{\S}$ & JAL 6703 \\
\hline & Erechtites valerianifolius (Wolf) DC. $\$$ & JAL 7347 \\
\hline & Eremanthus erythropappus (DC.) MacLeish & JAL 6814 \\
\hline & Erigeron maximus (D.Don) Otto ex DC. ${ }^{\S}$ & JAL 6674 \\
\hline & Galinsoga parviflora Cav. ${ }^{\S}$ & JAL 7402 \\
\hline & Gamochaeta americana (Mill.) Wedd. ${ }^{\S}$ & JAL 7086 \\
\hline & Gochnatia polymorpha (Less.) Cabrera ${ }^{1,2,3}$ & \\
\hline & Grazielia intermedia (DC.) R.M.King \& H.Rob. & JAL 7277 \\
\hline & Heterocondylus alatus (Vell.) R.M.King \& H.Rob. & JAL 6768 \\
\hline
\end{tabular}




\begin{tabular}{|c|c|c|}
\hline Família & Espécie & Voucher \\
\hline & Jaegeria hirta (Lag.) Less. ${ }^{\S}$ & JAL 6991 \\
\hline & Lepidaploa eriolepis (Gardner) H. Rob. & JAL 6803 \\
\hline & Mikania aff. cordifolia (L.f.) Willd. & JAL 6593 \\
\hline & M. erioclada DC. & JAL 6800 \\
\hline & M. glomerata Spreng. & JAL 6788 \\
\hline & M. lasiandrae DC. & JAL 6770 \\
\hline & M. micrantha Kunth & JAL 6771 \\
\hline & Mutisia coccinea A.St.-Hil. & JAL 6725 \\
\hline & Piptocarpha angustifolia Dusén ex Malme $\mathrm{e}^{1,2}$ & \\
\hline & P. axillaris (Less.) Baker & JAL 6836 \\
\hline & P. macropoda (DC.) Baker & JAL 6815 \\
\hline & P. sellowii (Sch.Bip.) Baker ${ }^{2}$ & \\
\hline & Podocoma notobellidiastrum (Griseb.) G.L.Nesom & JAL 6893 \\
\hline & Pterocaulon alopecuroides (Lam.) DC. $\S$ & JAL 7242 \\
\hline & Senecio brasiliensis (Spreng.) Less. & JAL 6853 \\
\hline & S. icoglossus DC. & JAL 6767 \\
\hline & S. oxyphyllus A. Cunn. ex DC. & JAL 6920 \\
\hline & Solidago chilensis Meyer ${ }^{\S}$ & JAL 7350 \\
\hline & Stevia collina Gardner & JAL 7346 \\
\hline & Symphyopappus reticulatus Baker & JAL 7266 \\
\hline & Tagetes minuta $\mathrm{L} . \S$ & JAL 6644 \\
\hline & Tilesia baccata (L.f.) Pruski & JAL 7283 \\
\hline & Tithonia diversifolia (Hemsl.) A.Gray ${ }^{\#}$ & JAL 6739 \\
\hline & Trichogoniopsis adenantha (DC.) R.M.King \& H.Rob. & JAL 6646 \\
\hline & Trixis antimenorrhoea (Schrank) Kuntze & JAL 6776 \\
\hline & T. praestans (Vell.) Cabrera & JAL 6844 \\
\hline & Vernonanthura discolor (Spreng.) H.Rob. ${ }^{2}$ & \\
\hline & V. divaricata (Spreng.) H.Rob. & JAL 6774 \\
\hline & V. petiolaris (DC.) H. Rob. & JAL 6595 \\
\hline & V. phosphorica (Vell.) H. Rob. & JAL 6838 \\
\hline & Viguiera arenaria Baker & JAL 7341 \\
\hline Balanophoraceae & Scybalium fungiforme Schott \& Endl. & JAL 7435 \\
\hline Balsaminaceae & Impatiens walleriana Hook.f. $\S$ & JAL 6673 \\
\hline \multirow[t]{4}{*}{ Begoniaceae } & Begonia angulata Vell. & JAL 7360 \\
\hline & B. cucullata Willd. & JAL 7055 \\
\hline & B. fischeri Schrank & JAL 6675 \\
\hline & B. parilis Irmsch. & JAL 6676 \\
\hline \multirow{5}{*}{$\begin{array}{l}\text { Bignoniaceae (R.G. } \\
\text { Udulutsch - HRCB) }\end{array}$} & Adenocalymma bracteatum (Cham.) DC. & JAL 6750 \\
\hline & A. marginatum (Cham.) DC. & JAL 7069 \\
\hline & Amphilophium dolichoides (Cham.) L.G.Lohmann & JAL 6951 \\
\hline & Dolichandra unguis-cati (L.) L.G.Lohmann & JAL 6648 \\
\hline & Fridericia formosa (Bureau) L.G.Lohmann & JAL 7014 \\
\hline
\end{tabular}




\begin{tabular}{|c|c|c|}
\hline Família & Espécie & Voucher \\
\hline & F. speciosa Mart. & JAL 7019 \\
\hline & Handroanthus albus (Cham.) Mattos ${ }^{1,2}$ & \\
\hline & H. chrysotrichus (Mart. ex DC.) Mattos ${ }^{1,2,3}$ & \\
\hline & H. impetiginosus Mattos $^{2}$ & \\
\hline & H. vellosoi (Toledo) Mattos $^{2}$ & \\
\hline & Jacaranda micrantha Cham. ${ }^{2,3}$ & \\
\hline & Jacaranda macrantha Cham. & JAL 7477 \\
\hline & Lundia corymbifera (Vahl) Sandwith & JAL 7353 \\
\hline & Mansoa difficilis (Cham.) Bureau \& K.Schum. & JAL 6718 \\
\hline & Mansoa sp. 1 & JAL 7012 \\
\hline & Pyrostegia venusta (Ker Gawl.) Miers & JAL 6787 \\
\hline & Stizophyllum perforatum (Cham.) Miers & JAL 6713 \\
\hline & Tanaecium selloi (Spreng.) L.G.Lohmann & JAL 6630 \\
\hline & Tynanthus micranthus Corr.Mello ex K.Schum. & JAL 6990 \\
\hline & Zeyheria tuberculosa (Vell.) Bureau ex Verl. ${ }^{2}$ & \\
\hline \multirow{6}{*}{$\begin{array}{l}\text { Blechnaceae (A. Salino - } \\
\text { BHCB) }\end{array}$} & Blechnum binervatum subsp. acutum (Desv.) R.M.Tryon \& Stolze & JAL 6828 \\
\hline & B. brasiliense Desv. & JAL 6747 \\
\hline & B. cordatum (Desv.) Hieron. & JAL 6748 \\
\hline & B. divergens Mett. & JAL 6721 \\
\hline & B. polypodioides Raddi & JAL 6655 \\
\hline & B. cf. usterianum (Christ.) C.Chr. & JAL 6772 \\
\hline \multirow[t]{6}{*}{ Boraginaceae } & Cordia ecalyculata Vell. ${ }^{2}$ & \\
\hline & C. polycephala (Lam.) I.M.Johnst. & JAL 7374 \\
\hline & C. sellowiana Cham. & JAL 6811 \\
\hline & C. trichotoma (Vell.) Arráb. ex Steud. ${ }^{1,2}$ & \\
\hline & Heliotropium transalpinum Vell. & JAL 7071 \\
\hline & Tournefortia paniculata Cham. & JAL 6835 \\
\hline Brassicaceae & Lepidium virginicum $\mathrm{L} . \S$ & JAL 7068 \\
\hline \multirow[t]{7}{*}{ Bromeliaceae } & Aechmea bromeliifolia (Rudge) Baker & JAL 7469 \\
\hline & A. distichantha Lem. & JAL 6669 \\
\hline & Ananas bracteatus (Lindl.) Schult. \& Schult.f. & JAL 6966 \\
\hline & Billbergia distachia (Vell.) Mez & JAL 6682 \\
\hline & Tillandsia gardneri Lindl. & JAL 6946 \\
\hline & T. recurvata (L.) L. & JAL 7343 \\
\hline & Vriesea carinata Wawra & JAL 7415 \\
\hline Burseraceae & Protium heptaphyllum (Aubl.) Marchand ${ }^{2,3}$ & \\
\hline \multirow[t]{6}{*}{ Cactaceae } & Cereus hildmannianus K.Schum. & JAL 6997 \\
\hline & Lepismium houlletianum (Lem.) Barthlott & JAL 6928 \\
\hline & Pereskia grandifolia Haw. & JAL 7511 \\
\hline & Rhipsalis floccosa Salm-Dyck ex Pfeiff. & JAL 7474 \\
\hline & R. paradoxa (Salm-Dyck ex Pfeiff.) Salm-Dyck & JAL 6904 \\
\hline & R. puniceodiscus G.Lindb. & JAL 6681 \\
\hline
\end{tabular}




\begin{tabular}{|c|c|c|}
\hline Família & Espécie & Voucher \\
\hline & R. teres (Vell.) Steud. & JAL 7416 \\
\hline \multirow[t]{2}{*}{ Campanulaceae } & Lobelia exaltata Pohl & JAL 6653 \\
\hline & Siphocampylus sulfureus E.Wimm. & JAL 6663 \\
\hline \multirow[t]{2}{*}{ Cannabaceae } & Celtis iguanaea (Jacq.) Sarg. & JAL 7009 \\
\hline & Trema micrantha (L.) Blume & JAL 6986 \\
\hline Cannaceae & Canna paniculata Ruiz \& Pav. & JAL 6773 \\
\hline Cardiopteridaceae & Citronella paniculata (Mart.) R.A.Howard ${ }^{1,2}$ & \\
\hline Caricaceae & Jacaratia spinosa (Aubl.) A.DC. ${ }^{2}$ & \\
\hline Caryophyllaceae & Drymaria cordata (L.) Willd. ex Roem. \& Schult. ${ }^{\S}$ & JAL 6978 \\
\hline \multirow[t]{8}{*}{ Celastraceae } & Hippocratea volubilis $\mathrm{L}$. & JAL 6923 \\
\hline & Maytenus aquifolia Mart. & JAL 6831 \\
\hline & M. evonymoides Reissek & JAL 6874 \\
\hline & M. gonoclada Mart. ${ }^{1,2}$ & \\
\hline & M. robusta Reissek & JAL 6902 \\
\hline & M. salicifolia Reissek & JAL 6666 \\
\hline & Peritassa hatschbachii Lombardi & JAL 7015 \\
\hline & Pristimera celastroides (Kunth) A.C.Sm. & JAL 6950 \\
\hline Chrysobalanaceae & Hirtella hebeclada Moric. ex DC. & JAL 6940 \\
\hline Clethraceae & Clethra scabra Pers. & JAL 6699 \\
\hline \multirow[t]{4}{*}{ Clusiaceae } & Calophyllum brasiliense Cambess. ${ }^{2}$ & \\
\hline & ${\text { Clusia criuva } \text { Cambess. }^{2}}^{2}$ & \\
\hline & Garcinia gardneriana (Planch. \& Triana) Zappi ${ }^{2}$ & \\
\hline & Tovomitopsis paniculata (Spreng.) Planch. \& Triana & JAL 6656 \\
\hline Combretaceae & Terminalia glabrescens Mart..$^{2,3}$ & \\
\hline \multirow[t]{5}{*}{ Commelinaceae } & Commelina sp. 1 & JAL 6760 \\
\hline & Tradescantia fluminensis Vell. & JAL 6981 \\
\hline & T. pallida (Rose) D.R.Hunt & JAL 7413 \\
\hline & T. zanonia (L.) Sw. & JAL 6829 \\
\hline & Tripogandra diuretica (Mart.) Handlos & JAL 7257 \\
\hline \multirow[t]{2}{*}{ Connaraceae } & Bernardinia fluminensis (Gardner) Planch. & JAL 6857 \\
\hline & Connarus regnellii G.Schellenb. & JAL 6654 \\
\hline \multirow[t]{6}{*}{ Convolvulaceae } & Dichondra microcalyx (Hallier f.) Fabris ${ }^{\S}$ & JAL 7263 \\
\hline & Ipomoea alba $\mathrm{L}$ & JAL 7093 \\
\hline & I. indica (Burm.) Merr. & JAL 6686 \\
\hline & Jacquemontia ciliata Sandwith & JAL 6623 \\
\hline & Merremia macrocalyx (Ruiz \& Pav.) O’Donell & JAL 7371 \\
\hline & M. umbellata (L.) Hallier f. & JAL 7338 \\
\hline Costaceae & Costus spiralis (Jacq.) Roscoe & JAL 7351 \\
\hline \multirow[t]{2}{*}{ Crassulaceae } & Bryophyllum delagoense (Eckl. \& Zeyh.) Druce & JAL 6638 \\
\hline & Sedum multiceps Coss. \& Durieu ${ }^{\S}$ & JAL 7501 \\
\hline \multirow[t]{2}{*}{ Cucurbitaceae } & Cayaponia diversifolia (Cogn.) Cogn. & JAL 6793 \\
\hline & Cayaponia sp. 1 & JAL 7389 \\
\hline
\end{tabular}




\begin{tabular}{|c|c|c|}
\hline Família & Espécie & Voucher \\
\hline & Melothria cucumis Vell. & JAL 7342 \\
\hline & Wilbrandia hibiscoides Silva Manso & JAL 6862 \\
\hline & W. longibracteata Cogn. & JAL 6939 \\
\hline & Indet. 1 & JAL 7294 \\
\hline & Indet. 2 & JAL 7386 \\
\hline Cunoniaceae & Lamanonia ternata Vell. (=Lamanonia speciosa (Cambess.) L.B.Sm.) & JAL 6473 \\
\hline Cupressaceae & Cupressus lusitanica Mill." & JAL 6889 \\
\hline \multirow{3}{*}{$\begin{array}{l}\text { Cyatheaceae (T.E. Almeida, } \\
\text { A. Salino - BHCB) }\end{array}$} & Alsophila setosa Kaulf. & JAL 6639 \\
\hline & Cyathea atrovirens (Langsd. \& Fisch.) Domin & JAL 6668 \\
\hline & C. delgadii Sternb. & \\
\hline \multirow{19}{*}{$\begin{array}{l}\text { Cyperaceae (S. Martins - } \\
\text { HRCB, M.V. Alves - UFP) }\end{array}$} & Bolboschoenus robustus (Pursh) Soják & JAL 6778 \\
\hline & Bulbostylis capillaris (L.) C.B.Clarke & JAL 6947 \\
\hline & Carex pseudocyperus L. $\S$ & JAL 6933 \\
\hline & Cyperus friburgensis Boeckeler & JAL 7039 \\
\hline & C. haspan $\mathrm{L}$. & JAL 6931 \\
\hline & C. cf. luzulae (L.) Retz. & JAL 6932 \\
\hline & Cyperus sp. 1 & JAL 7037 \\
\hline & Cyperus sp. 2 & JAL 7363 \\
\hline & Cyperus sp. 3 & JAL 7376 \\
\hline & Eleocharis sp. 1 & JAL 7026 \\
\hline & Fimbristylis autumnalis (L.) Roem. \& Schult. & JAL 7084 \\
\hline & Pleurostachys foliosa Kunth & JAL 6885 \\
\hline & P. stricta Kunth & JAL 7268 \\
\hline & Rhynchospora corymbosa (L.) Britton & JAL 6649 \\
\hline & R. cf. holoschoenoides (Rich.) Herter & JAL 7075 \\
\hline & R. splendens Lindm. & JAL 6779 \\
\hline & Scleria myriocarpa Kunth & JAL 6810 \\
\hline & S. panicoides Kunth & JAL 6667 \\
\hline & S. variegata (Nees) Steud. & JAL 6808 \\
\hline $\begin{array}{l}\text { Dicksoniaceae (T.E. Almeida } \\
\text { - BHCB) }\end{array}$ & Dicksonia sellowiana Hook. & JAL 8651 \\
\hline \multirow{3}{*}{$\begin{array}{l}\text { Dioscoreaceae (D. Araújo - } \\
\text { HRCB) }\end{array}$} & Dioscorea fodinarum Kunth & JAL 6929 \\
\hline & D. multiflora Mart. ex Griseb. & JAL 7092 \\
\hline & D. subhastata Vell. & JAL 7356 \\
\hline \multirow{6}{*}{$\begin{array}{l}\text { Dryopteridaceae (A. Salino, } \\
\text { L.C.N. Melo-BHCB) }\end{array}$} & Ctenitis submarginalis (Langsd. \& Fisch.) Ching & JAL 6830 \\
\hline & Didymochlaena truncatula (Sw.) J.Sm. & JAL 6925 \\
\hline & Elaphoglossum lingua (C.Presl) Brack. & JAL 7315 \\
\hline & Megalastrum sp. & JAL 8657 \\
\hline & Polybotrya cylindrica Kaulf. & JAL 7043 \\
\hline & Rumohra adiantiformis (G.Forst.) Ching & JAL 6722 \\
\hline Ebenaceae & Diospyros inconstans Jacq. & JAL 7506 \\
\hline
\end{tabular}




\begin{tabular}{|c|c|c|}
\hline Família & Espécie & Voucher \\
\hline Elaeocarpaceae & Sloanea hirsuta (Schott) Planch. ex Benth. & JAL 8640 \\
\hline \multirow[t]{2}{*}{ Erythroxylaceae } & Erythroxylum daphnites Mart. & JAL 6926 \\
\hline & E. deciduum A.St.-Hil. & JAL 6456 \\
\hline \multirow[t]{23}{*}{ Euphorbiaceae } & Acalypha chamaedrifolia (Lam.) Müll.Arg." & JAL 6955 \\
\hline & Actinostemon concolor (Spreng.) Müll.Arg. & JAL 7463 \\
\hline & A. klotzschii (Didr.) Pax & JAL 6869 \\
\hline & Alchornea glandulosa subsp. iricurana (Casar.) Secco & JAL 6743 \\
\hline & A. sidifolia Müll.Arg. ${ }^{2}$ & \\
\hline & A. triplinervia (Spreng.) Müll.Arg. ${ }^{1,2,3}$ & \\
\hline & Aparisthmium cordatum (A.Juss.) Baill. & JAL 7382 \\
\hline & Bia alienata Didr. & JAL 7011 \\
\hline & Croton floribundus Spreng. & JAL 6976 \\
\hline & C. lundianus (Didr.) Müll.Arg. & JAL 6927 \\
\hline & C. macrobothrys Baill. & JAL 6971 \\
\hline & C. salutaris Casar. $^{2}$ & \\
\hline & C. urucurana Baill. & JAL 7089 \\
\hline & Dalechampia triphylla Lam. & JAL 6818 \\
\hline & Euphorbia cotinifolia L." & JAL 7087 \\
\hline & E. milii Des Moul." & JAL 7493 \\
\hline & E. pulcherrima Willd. ex Klotzsch ${ }^{\#}$ & JAL 6728 \\
\hline & Ricinus communis L. $\S$ & JAL 6961 \\
\hline & Sapium glandulosum (L.) Morong & JAL 6942 \\
\hline & Sebastiania edwalliana Pax \& K.Hoffm. & LBS 83 \\
\hline & S. serrata Müll.Arg. ${ }^{1,3}$ & \\
\hline & Indet. 1 & JAL 7316 \\
\hline & Indet. 2 & JAL 6890 \\
\hline \multirow{16}{*}{$\begin{array}{l}\text { Fabaceae (L.P. Queiroz - } \\
\text { HUEFS) }\end{array}$} & Aeschynomene sp. 1 & JAL 7018 \\
\hline & Albizia pedicellaris (DC.) L. Rico ${ }^{2}$ & \\
\hline & A. polycephala (Benth.) Killip ex Record ${ }^{2}$ & \\
\hline & Anadenanthera colubrina (Vell.) Brenan & JAL 7003 \\
\hline & A. peregrina (L.) Speg. ${ }^{2}$ & \\
\hline & Andira fraxinifolia Benth. & JAL 6891 \\
\hline & A. inermis (W.Wright) DC. ${ }^{2,3}$ & \\
\hline & Bauhinia forficata Link $^{2}$ & \\
\hline & B. longifolia (Bong.) Steud. & JAL 7085 \\
\hline & Calliandra brevipes Benth." & JAL 7468 \\
\hline & C. tweedii Benth." & JAL 7475 \\
\hline & Cassia ferruginea (Schrad.) Schrad. ex DC. & JAL 7002 \\
\hline & Centrolobium tomentosum Benth. ${ }^{2}$ & \\
\hline & Chamaecrista flexuosa (L.) Greene ${ }^{\S}$ & JAL 7344 \\
\hline & Copaifera langsdorffii Desf. ${ }^{1,2,3}$ & \\
\hline & Crotalaria breviflora DC. & JAL 7240 \\
\hline
\end{tabular}




\begin{tabular}{|c|c|c|}
\hline Família & Espécie & Voucher \\
\hline & C. incana $\mathrm{L} . \S$ & JAL 6726 \\
\hline & C. lanceolata E.Mey & JAL 6738 \\
\hline & C. micans Link $^{\S}$ & JAL 7017 \\
\hline & Dalbergia brasiliensis Vogel & JAL 7038 \\
\hline & D. frutescens (Vell.) Britton ${ }^{1}$ & \\
\hline & D. villosa (Benth.) Benth., ${ }^{2,3}$ & \\
\hline & Desmodium discolor Vogel $^{\S}$ & JAL 7326 \\
\hline & D. incanum DC. $\$$ & JAL 6858 \\
\hline & Dioclea rufescens Benth. & JAL 7510 \\
\hline & Enterolobium contortisiliquum (Vell.) Morong ${ }^{2}$ & \\
\hline & Erythrina falcata Benth. $^{2}$ & \\
\hline & E. speciosa Andrews ${ }^{\#}$ & JAL 7452 \\
\hline & Holocalyx balansae Micheli ${ }^{2}$ & \\
\hline & Hymenaea courbaril L. ${ }^{2}$ & \\
\hline & Inga laurina (Sw.) Willd. ${ }^{2}$ & \\
\hline & I. marginata Willd. & JAL 7081 \\
\hline & I. sessilis (Vell.) Mart. & JAL 6466 \\
\hline & I. striata Benth. & JAL 7483 \\
\hline & I. subnuda Salzm. ex Benth. ${ }^{2}$ & \\
\hline & I. vera Willd. $^{2}$ & \\
\hline & Leucochloron incuriale (Vell.) Barneby \& J.W.Grimes & JAL 6864 \\
\hline & Lonchocarpus campestris Mart. ex Benth., & \\
\hline & L. muehlbergianus Hassl. & JAL 6724 \\
\hline & L. subglaucescens Mart. ex Benth ${ }^{2,3}$ & \\
\hline & Luetzelburgia guaissara Toledo² & \\
\hline & Machaerium brasiliense Vogel & JAL 6918 \\
\hline & M. lanceolatum (Vell.) J.F.Macbr. ${ }^{1}$ & \\
\hline & M. nictitans (Vell.) Benth. & JAL 7372 \\
\hline & M. oblongifolium Vogel & JAL 7524 \\
\hline & M. scleroxylon Tul. ${ }^{2}$ & \\
\hline & M. stipitatum (DC.) Vogel ${ }^{2,3}$ & \\
\hline & M. villosum Vogel & JAL 6765 \\
\hline & Mucuna pruriens (L.) DC. & JAL 7325 \\
\hline & Myrocarpus frondosus Allemão ${ }^{1}$ & \\
\hline & Myroxylon peruiferum L.f. ${ }^{2}$ & \\
\hline & Ormosia arborea (Vell.) Harms & JAL 7318 \\
\hline & O. minor Vogel ${ }^{1,2}$ & \\
\hline & Parapiptadenia rigida (Benth.) Brenan ${ }^{2}$ & \\
\hline & Peltophorum dubium (Spreng.) Taub. ${ }^{2}$ & \\
\hline & Piptadenia gonoacantha (Mart.) J.F.Macbr. ${ }^{2,3}$ & \\
\hline & Platymiscium floribundum Vogel & JAL 7495 \\
\hline & Platypodium elegans Vogel $^{2}$ & \\
\hline
\end{tabular}




\begin{tabular}{|c|c|c|}
\hline Família & Espécie & Voucher \\
\hline & Pterocarpus rohrii Vahl $^{2}$ & \\
\hline & Pterogyne nitens Tul. ${ }^{2}$ & \\
\hline & Schizolobium parahyba (Vell.) Blake ${ }^{2}$ & \\
\hline & Senegalia polyphylla (DC.) Britton \& Rose $\mathrm{R}^{2,3}$ & \\
\hline & Senegalia sp. 1 & JAL 7302 \\
\hline & Senna pendula (Humb.\&Bonpl. ex Willd.) H.S.Irwin \& Barneby & JAL 6685 \\
\hline & S. splendida (Vogel) H.S.Irwin \& Barneby & JAL 6611 \\
\hline & Senna sp. 1 & JAL 7512 \\
\hline & Stylosanthes guianensis (Aubl.) Sw. ${ }^{\S}$ & JAL 6715 \\
\hline & Sweetia fruticosa Spreng. $^{2}$ & \\
\hline & Vigna latidenticulata (Harms) A.Delgado ${ }^{\S}$ & JAL 7297 \\
\hline & Vigna sp. $1^{\S}$ & JAL 6601 \\
\hline & Zollernia ilicifolia (Brongn.) Vogel ${ }^{2}$ & \\
\hline & Zornia gemella Vogel ${ }^{\S}$ & JAL 7053 \\
\hline Geraniaceae & Pelargonium hortorum L.H.Bailey ${ }^{\#}$ & JAL 6960 \\
\hline \multirow{4}{*}{$\begin{array}{l}\text { Gesneriaceae (A. Chautems } \\
-\mathrm{G})\end{array}$} & Nematanthus striatus (Handro) Chautems & JAL 6684 \\
\hline & Sinningia aggregata (Ker Gawl.) Wiehler & JAL 6916 \\
\hline & S. allagophylla (Mart.) Wiehler & JAL 7509 \\
\hline & S. douglasii (Lindl.) Chautems & JAL 6915 \\
\hline \multirow{2}{*}{$\begin{array}{l}\text { Gleicheniaceae (A. Salino - } \\
\text { BHCB) }\end{array}$} & Sticherus lanuginosus (Fée) Nakai & JAL 6693 \\
\hline & Sticherus sp. 1 & JAL 7273 \\
\hline Heliconiaceae & Heliconia rostrata Ruiz \& Pav. & JAL 7329 \\
\hline \multirow[t]{2}{*}{ Hypericaceae } & Hypericum brasiliense Choisy & JAL 7079 \\
\hline & Vismia micrantha A.St.-Hil. & JAL 7256 \\
\hline Hypoxidaceae & Hypoxis decumbens L.§ & JAL 7021 \\
\hline \multirow[t]{4}{*}{ Iridaceae } & Crocosmia crocosmiiflora (Lemoine ex Morren) N.E.Br. ${ }^{\S}$ & JAL 6680 \\
\hline & Neomarica candida (Hassl.) Sprague & JAL 6873 \\
\hline & Sisyrinchium micranthum Cav. & JAL 6954 \\
\hline & Trimezia martinicensis (Jacq.) Herb. & JAL 6952 \\
\hline Juncaceae & Juncus microcephalus Kunth & JAL 6618 \\
\hline \multirow[t]{11}{*}{ Lamiaceae } & Aegiphila integrifolia (Jacq.) Moldenke & JAL 7285 \\
\hline & Eriope macrostachya Mart. ex Benth. ${ }^{\S}$ & JAL 6993 \\
\hline & Hyptis mutabilis (Rich.) Briq. ${ }^{\S}$ & JAL 6683 \\
\hline & Leonurus sibiricus L. & JAL 7062 \\
\hline & Ocimum carnosum (Spreng.) Link \& Otto ex Benth. ${ }^{\S}$ & JAL 7239 \\
\hline & Peltodon radicans Pohl & JAL 6631 \\
\hline & Plectranthus barbatus Andrews ${ }^{\#}$ & JAL 6842 \\
\hline & P. neochilus Schltr. ${ }^{\S}$ & JAL 6905 \\
\hline & Salvia coccinea Buc’hoz ex Etl. ${ }^{\S}$ & JAL 7064 \\
\hline & Tetradenia riparia (Hochst.) Codd ${ }^{\#}$ & JAL 7428 \\
\hline & Vitex megapotamica (Spreng.) Moldenke & JAL 7040 \\
\hline
\end{tabular}




\begin{tabular}{|c|c|c|}
\hline Família & Espécie & Voucher \\
\hline & V. polygama Cham. & JAL 7035 \\
\hline & Indet. 1 & JAL 7420 \\
\hline \multirow{25}{*}{$\begin{array}{l}\text { Lauraceae (P.L.R. Moraes - } \\
\text { HUEFS) }\end{array}$} & Aniba firmula (Nees \& Mart.) Mez & JAL 7445 \\
\hline & Cinnamomum stenophyllum (Meisn.) Vattimo-Gil ${ }^{1,2,3}$ & \\
\hline & C. triplinerve (Ruiz \& Pav.) Kosterm. ${ }^{1}$ & \\
\hline & Cryptocarya aschersoniana $\mathrm{Mez}^{1,2}$ & \\
\hline & C. moschata Nees \& Mart. ${ }^{2,3}$ & \\
\hline & Endlicheria paniculata (Spreng.) J.F.Macbr. & JAL 7307 \\
\hline & Nectandra grandiflora $\mathrm{Nees}^{2,3}$ & \\
\hline & N. megapotamica (Spreng.) Mez & JAL 6917 \\
\hline & N. oppositifolia $\mathrm{Nees}^{1,2}$ & \\
\hline & Ocotea bicolor Vattimo-Gil ${ }^{1}$ & \\
\hline & O. corymbosa (Meisn.) Mez $z^{1,2,3}$ & \\
\hline & O. diospyrifolia (Meisn.) $\mathrm{Mez}^{2}$ & \\
\hline & O. elegans $\mathrm{Mez}^{2,3}$ & \\
\hline & O. glaziovii $\mathrm{Mez}^{2}$ & \\
\hline & O. lanata (Nees \& Mart.) $\mathrm{Mez}^{2}$ & \\
\hline & O. minarum (Nees \& Mart.) Mez & JAL 6973 \\
\hline & O. nutans (Nees) Mez & JAL 6457 \\
\hline & O. odorifera (Vell.) Rohwer ${ }^{2}$ & \\
\hline & O. puberula (Rich.) Nees ${ }^{1,2,3}$ & \\
\hline & O. pulchella (Nees \& Mart.) Mez ${ }^{2}$ & \\
\hline & O. silvestris Vattimo-Gil ${ }^{1}$ & \\
\hline & O. teleiandra (Meisn.) $\mathrm{Mez}^{3}$ & \\
\hline & Persea americana Mill. ${ }^{\S}$ & JAL 7449 \\
\hline & P. venosa Nees \& Mart. ${ }^{1,2,3}$ & \\
\hline & P. willdenovii Kosterm. & JAL 7287 \\
\hline Laxmanniaceae & Cordyline sp. 1 & JAL 6944 \\
\hline \multirow[t]{2}{*}{ Lecythidaceae } & Cariniana estrellensis (Raddi) Kuntze $\mathrm{e}^{1,2,3}$ & \\
\hline & C. legalis (Mart.) Kuntze ${ }^{2}$ & \\
\hline \multirow[t]{3}{*}{ Loganiaceae } & Spigelia beyrichiana Cham. \& Schltdl. & JAL 6912 \\
\hline & Spigelia sp. 1 & JAL 6909 \\
\hline & Strychnos brasiliensis Mart. & JAL 6957 \\
\hline $\begin{array}{l}\text { Lomariopsidaceae (A. Salino } \\
\text { - BHCB) }\end{array}$ & Nephrolepis cordifolia (L.) C.Presl & JAL 7395 \\
\hline \multirow[t]{3}{*}{ Loranthaceae } & Struthanthus flexicaulis Mart. & JAL 6695 \\
\hline & S. marginatus (Desr.) Blume & JAL 6843 \\
\hline & S. staphylinus Mart. & JAL 7479 \\
\hline \multirow{2}{*}{$\begin{array}{l}\text { Lycopodiaceae (A. Salino - } \\
\text { BHCB) }\end{array}$} & Lycopodiella cernua (L.) Pic.Serm. & JAL 6599 \\
\hline & Lycopodium thyoides Humb. \& Bonpl. ex Willd. & JAL 7441 \\
\hline Lythraceae & Cuphea calophylla subsp. mesostemon (Koehne) Lourteig & JAL 6979 \\
\hline
\end{tabular}




\begin{tabular}{|c|c|c|}
\hline Família & Espécie & Voucher \\
\hline & Lafoensia pacari A.St.-Hil. & JAL 7023 \\
\hline \multirow[t]{2}{*}{ Magnoliaceae } & Magnolia champaca (L.) Baill. ex Pierre ${ }^{\#}$ & JAL 7050 \\
\hline & M. ovata (A.St.-Hil.) Spreng. ${ }^{2}$ & \\
\hline \multirow{10}{*}{$\begin{array}{l}\text { Malpighiaceae (M.C.H. } \\
\text { Mamede - SP) }\end{array}$} & Alicia anisopetala (A.Juss.) W.R.Anderson & JAL 6733 \\
\hline & Heteropterys intermedia (A.Juss.) Griseb. & JAL 6742 \\
\hline & H. umbellata A.Juss. & JAL 7088 \\
\hline & Mascagnia cordifolia (A.Juss.) Griseb. & JAL 7052 \\
\hline & Mascagnia sp. 1 & JAL 6717 \\
\hline & Niedenzuella acutifolia (Cav.) W.R.Anderson & JAL 7251 \\
\hline & Peixotoa parviflora A.Juss. & JAL 6636 \\
\hline & Tetrapterys phlomoides (Spreng.) Nied. & JAL 6650 \\
\hline & Indet. 1 & JAL 7387 \\
\hline & Indet. 2 & JAL 7462 \\
\hline \multirow{21}{*}{$\begin{array}{l}\text { Malvaceae (A. Krapovickas } \\
\text { - CTES) }\end{array}$} & Abutilon bedfordianum (Hook.) A.St.-Hil. \& Naudin & JAL 6746 \\
\hline & Bastardiopsis densiflora (Hook. \& Arn.) Hassl. ${ }^{2}$ & \\
\hline & Ceiba speciosa (A.St.-Hil.) Ravenna & JAL 7270 \\
\hline & Eriotheca candolleana (K.Schum.) A.Robyns ${ }^{2}$ & \\
\hline & Guazuma ulmifolia Lam. $^{2}$ & \\
\hline & Helicteres ovata Lam. $^{2}$ & \\
\hline & Heliocarpus popayanensis $\mathrm{Kunth}^{2}$ & \\
\hline & Luehea divaricata Mart. \& Zucc. ${ }^{2}$ & \\
\hline & L. grandiflora Mart. \& Zucc. & JAL 6854 \\
\hline & L. paniculata Mart. \& Zucc. ${ }^{2}$ & \\
\hline & Malvaviscus arboreus Cav." & JAL 7401 \\
\hline & Pavonia communis A.St.-Hil. & JAL 6938 \\
\hline & P. nemoralis A.St.-Hil. \& Naudin & JAL 7253 \\
\hline & P. sepium A.St.-Hil. & JAL 7262 \\
\hline & Pseudobombax grandiflorum (Cav.) A.Robyns & JAL 6720 \\
\hline & P. longiflorum (Mart. \& Zucc.) A.Robyns ${ }^{2}$ & \\
\hline & Sida sp.nov. & JAL 6633 \\
\hline & Triumfetta semitriloba Jacq. & JAL 6634 \\
\hline & Waltheria americana $\mathrm{L} . \S$ & JAL 6702 \\
\hline & Wissadula hernandioides (L.Hér.) Garcke & JAL 7330 \\
\hline & W. parviflora (A.St.-Hil.) R.E.Fr. & JAL 6621 \\
\hline \multirow[t]{2}{*}{ Marantaceae } & Calathea cf. monophylla (Vell.) Körn. & JAL 7236 \\
\hline & Ctenanthe lanceolata Petersen & JAL 7076 \\
\hline \multirow{4}{*}{$\begin{array}{l}\text { Melastomataceae (R. } \\
\text { Goldenberg - UPCB, G.A.R. } \\
\text { Silveira - UEC) }\end{array}$} & Leandra aurea (Cham.) Cogn. & JAL 6775 \\
\hline & L. melastomoides Raddi & JAL 6619 \\
\hline & L. purpurascens (DC.) Cogn. & JAL 6841 \\
\hline & Miconia cinnamomifolia (DC.) Naudin ${ }^{1,2}$ & \\
\hline
\end{tabular}




\begin{tabular}{|c|c|c|}
\hline Família & Espécie & Voucher \\
\hline & M. discolor DC. & JAL 6769 \\
\hline & M. ibaguensis (Bonpl.) Triana & JAL 6476 \\
\hline & M. latecrenata (DC.) Naudin & JAL 6632 \\
\hline & M. ligustroides (DC.) Naudin & JAL 6641 \\
\hline & M. pusilliflora (DC.) Naudin & JAL 6609 \\
\hline & M. selllowiana Naudin & JAL 6804 \\
\hline & Ossaea amygdaloides (DC.) Triana & JAL 6658 \\
\hline & O. marginata (Desr.) Triana & JAL 6878 \\
\hline & Tibouchina granulosa (Desr.) Cogn. & JAL 7247 \\
\hline & T. pulchra Cogn. ${ }^{2}$ & \\
\hline & T. sebastianopolitana (Raddi) Cogn. & JAL 6614 \\
\hline & T. sellowiana Cogn. & JAL 7423 \\
\hline & T. semidecandra (Schrank \& Mart. ex DC.) Cogn. & JAL 7439 \\
\hline & Tibouchina sp. 1 & JAL 6624 \\
\hline & Tibouchina sp. 2 & JAL 6762 \\
\hline \multirow[t]{12}{*}{ Meliaceae } & Cabralea canjerana (Vell.) Mart. & JAL 6736 \\
\hline & Cedrela fissilis Vell.., ${ }^{1,3}$ & \\
\hline & C. odorata $\mathrm{L}$. & JAL 7516 \\
\hline & Guarea guidonia (L.) Sleumer ${ }^{1,2}$ & \\
\hline & G. macrophylla Vahl & JAL 6970 \\
\hline & Trichilia casaretti C.DC. $^{2}$ & \\
\hline & T. catigua A.Juss. & JAL 7004 \\
\hline & T. clausseni $\mathrm{C}^{*}$.DC. ${ }^{2}$ & \\
\hline & T. elegans A.Juss. & JAL 6967 \\
\hline & T. hirta L. ${ }^{2}$ & \\
\hline & T. cf. pallens C.DC. ${ }^{1}$ & \\
\hline & T. pallida $\mathrm{Sw}$ & JAL 7403 \\
\hline \multirow[t]{4}{*}{ Menispermaceae } & Abuta selloana Eichler & JAL 6983 \\
\hline & Cissampelos andromorpha DC. & JAL 6465 \\
\hline & C. pareira $\mathrm{L}$. & JAL 7010 \\
\hline & Hyperbaena oblongifolia (Mart.) Chodat \& Hassl. & JAL 7421 \\
\hline \multirow[t]{4}{*}{ Monimiaceae } & Mollinedia argyrogyna Perkins & JAL 6907 \\
\hline & M. clavigera Tul. & JAL 6870 \\
\hline & M. schottiana (Spreng.) Perkins ${ }^{2}$ & \\
\hline & M. uleana Perkins ${ }^{2}$ & \\
\hline \multirow[t]{7}{*}{ Moraceae } & Ficus citrifolia Mill. ${ }^{2}$ & \\
\hline & F. enormis Mart. ex Miq. $^{2}$ & \\
\hline & F. eximia Schott $^{2}$ & \\
\hline & F. guaranitica Chodat $^{2}$ & \\
\hline & F. luschnathiana (Miq.)Miq. & JAL 7471 \\
\hline & F. pertusa L.f. $^{2}$ & \\
\hline & Morus nigra L.\# & JAL 6846 \\
\hline
\end{tabular}




\begin{tabular}{|c|c|c|}
\hline Família & Espécie & Voucher \\
\hline & Maclura tinctoria (L.) D. Don ex Steud. 2,3 & \\
\hline & Sorocea bonplandii (Baill.) W.C.Burger et al. & JAL 6812 \\
\hline \multirow{5}{*}{$\begin{array}{l}\text { Myrsinaceae (L.C. Bernacci } \\
- \text { IAC) }\end{array}$} & Cybianthus cuneifolius Mart. & JAL 7286 \\
\hline & Myrsine coriacea (Sw.) R.Br. ex Roem. \& Schult. & JAL 6706 \\
\hline & M. gardneriana A.DC. & JAL 6657 \\
\hline & M. lancifolia Mart. $^{2}$ & \\
\hline & M. umbellata Mart. & JAL 6472 \\
\hline \multirow{34}{*}{$\begin{array}{l}\text { Myrtaceae (M. Sobral - } \\
\text { BHCB) }\end{array}$} & Calyptranthes clusiifolia O.Berg ${ }^{2}$ & \\
\hline & C. concinna $\mathrm{DC} .^{2}$ & \\
\hline & Campomanesia guazumifolia (Cambess.) O.Berg..$^{1,2,3}$ & \\
\hline & C. maschalantha (O.Berg) Kiaersk. ${ }^{2}$ & \\
\hline & C. phaea (O. Berg) Landrum² & \\
\hline & Campomanesia sp. 1 & JAL 7507 \\
\hline & Eucalyptus sp. $1^{\S}$ & JAL 7460 \\
\hline & Eugenia acutata Miq. & JAL 7072 \\
\hline & E. biflora (L.) DC. ${ }^{2}$ & \\
\hline & E. blastantha (O.Berg) D.Legrand $d^{2,3}$ & \\
\hline & E. brasiliensis Lam. & JAL 7515 \\
\hline & E. florida $\mathrm{DC}^{2}$ & \\
\hline & E. geminiflora O.Berg ${ }^{2}$ & \\
\hline & E. handroana D.Legrand ${ }^{2}$ & \\
\hline & E. involucrata DC. ${ }^{2}$ & \\
\hline & E. kleinii D.Legrand & JAL 6477 \\
\hline & E. leptoclada O.Berg & JAL 7241 \\
\hline & E. ligustrina (Sw.) Willd. ${ }^{2}$ & \\
\hline & E. myrcianthes Nied. ${ }^{2}$ & \\
\hline & E. cf. neosilvestris Sobral & JAL 6837 \\
\hline & E. neolaurifolia. Sobral ${ }^{2,3}$ & \\
\hline & E. neomyrtifolia Sobral ${ }^{1,2}$ & \\
\hline & E. neoverrucosa Sobral & LBS 87 \\
\hline & E. prasina O.Berg. & \\
\hline & E. pyriformis Cambess. & JAL 6475 \\
\hline & E. speciosa Cambess. ${ }^{1,2}$ & \\
\hline & E. sulcata Spring ex Mart. $^{2}$ & JAL 7339 \\
\hline & E. tenuipedunculata Kiaersk. ${ }^{2,3}$ & \\
\hline & E. vattimoana Mattos ${ }^{2,3}$ & \\
\hline & E. verticillata (Vell.) Angely ${ }^{2}$ & \\
\hline & Eugenia sp. 1 & JAL 6714 \\
\hline & Marlierea silvatica (O.Berg.) Kiaersk..$^{2,3}$ & \\
\hline & Myrceugenia campestris (DC.) D.Legrand \& Kausel ${ }^{2,3}$ & \\
\hline & M. myrcioides (Cambess.) O.Berg ${ }^{2}$ & \\
\hline
\end{tabular}




\begin{tabular}{|c|c|c|}
\hline Família & Espécie & Voucher \\
\hline & Myrcia glazioviana Kiaersk. $^{2}$ & \\
\hline & M. guianensis (Aubl.) DC. ${ }^{2}$ & \\
\hline & M. hebepetala $\mathrm{DC} .^{2}$ & \\
\hline & M. multiflora (Lam.) DC. ${ }^{2}$ & \\
\hline & M. aff. retorta Cambess. ${ }^{1}$ & \\
\hline & M. rufipes DC. ${ }^{2}$ & \\
\hline & M. spectabilis $\mathrm{DC} .^{2}$ & \\
\hline & M. splendens (Sw.) DC. & JAL 6996 \\
\hline & M. tomentosa (Aubl.) DC. ${ }^{2}$ & \\
\hline & M. undulata O.Berg ${ }^{2}$ & \\
\hline & M. venulosa DC..$^{2,3}$ & \\
\hline & Myrciaria floribunda (H.West ex Willd.) O.Berg & JAL 7288 \\
\hline & Myrcianthes pungens (O.Berg) D.Legrand ${ }^{2}$ & \\
\hline & Pimenta pseudocaryophyllus (Gomes) Landrum ${ }^{1,2,3}$ & \\
\hline & Psidium cattleianum Sabine & JAL 7066 \\
\hline & P. grandifolium Mart. ex DC. ${ }^{2}$ & \\
\hline & Siphoneugena densiflora O.Berg ${ }^{1,2,3}$ & \\
\hline \multirow[t]{4}{*}{ Nyctaginaceae } & Bougainvillea spectabilis Willd. & JAL 7500 \\
\hline & Guapira opposita (Vell.) Reitz & JAL 6865 \\
\hline & Mirabilis jalapa L. $\S$ & JAL 7238 \\
\hline & Pisonia ambigua Heimerl & JAL 6866 \\
\hline \multirow[t]{3}{*}{ Ochnaceae } & Ouratea castaneifolia (DC.) Engl. ${ }^{2}$ & \\
\hline & O. parviflora (A.DC.) Baill. ${ }^{2}$ & \\
\hline & O. semiserrata (Mart. \& Nees) Engl. ${ }^{1,2,3}$ & \\
\hline \multirow[t]{3}{*}{ Onagraceae } & Fuchsia regia (Vell.) Munz & JAL 6903 \\
\hline & Ludwigia elegans (Cambess.) H.Hara & JAL 7078 \\
\hline & L. sericea (Cambess.) H.Hara & JAL 7359 \\
\hline Opiliaceae & Agonandra excelsa Griseb. & \\
\hline \multirow[t]{14}{*}{ Orchidaceae } & Acianthera aphthosa (Lindl.) Pridgeon \& M.W.Chase ${ }^{4}$ & \\
\hline & A. auriculata (Lindl.) Pridgeon \& M.W.Chase ${ }^{4}$ & \\
\hline & A. leptotifolia (Barb.Rodr.) Pridgeon \& M.W.Chase ${ }^{4}$ & \\
\hline & A. luteola (Lindl.) Pridgeon \& M.W.Chase ${ }^{4}$ & \\
\hline & A. saundersiana (Rchb.f.) Pridgeon \& M.W.Chase ${ }^{4}$ & \\
\hline & A. saurocephala (Lodd.) Pridgeon \& M.W.Chase ${ }^{4}$ & \\
\hline & Alatiglossum longipes (Lindl.) Baptista ${ }^{4}$ & \\
\hline & Aspidogyne hylibates (Rchb.f.) Garay ${ }^{4}$ & \\
\hline & A. metallescens (Barb.Rodr.) Garay ${ }^{4}$ & \\
\hline & Baptistonia fimbriata (Lindl.) Chiron \& V.P.Castro ${ }^{4}$ & \\
\hline & B. pubes (Lindl.) Chiron \& V.P.Castro ${ }^{4}$ & \\
\hline & B. sarcodes (Lindl.) Chiron \& V.P.Castro ${ }^{4}$ & \\
\hline & Barbosella cogniauxiana (Speg. \& Kraenzl.)Schltr. ${ }^{4}$ & \\
\hline & Bifrenaria harrisoniae (Hook.) Rchb.f. ${ }^{4}$ & \\
\hline
\end{tabular}




\begin{tabular}{|c|c|c|}
\hline Família & Espécie & Voucher \\
\hline & Brasilidium crispum (Lodd.) Campacci ${ }^{4}$ & \\
\hline & B. praetextum (Rchb.f.) Campacci ${ }^{4}$ & \\
\hline & Brasiliorchis chrysantha (Barb.Rodr.) R.B.Singer et al. ${ }^{4}$ & \\
\hline & B. consanguinea (Klotzsch) R.B.Singer et al. ${ }^{4}$ & \\
\hline & B. gracilis (Lodd.) R.B.Singer et al. ${ }^{4}$ & \\
\hline & B. picta (Hook.) R.B.Singer et al. ${ }^{4}$ & \\
\hline & Bulbophyllum chloroglossum Rchb.f. \& Warm. ${ }^{4}$ & \\
\hline & B. exaltatum Lindl. $^{4}$ & \\
\hline & B. glutinosum (Barb.Rodr.) Cogn. ${ }^{4}$ & \\
\hline & B. regnellii Rchb.f. ${ }^{4}$ & \\
\hline & Campylocentrum micranthum (Lindl.) Rolfe ${ }^{4}$ & \\
\hline & Capanemia superflua (Rchb.f.) Garay ${ }^{4}$ & \\
\hline & C. thereziae Barb.Rodr. ${ }^{4}$ & \\
\hline & Catasetum cernuum (Lindl.) Rchb.f. ${ }^{4}$ & \\
\hline & Cattleya loddigesii Lind.$^{4}$ & \\
\hline & Christensonella cepula (Rchb.f.) S.Koehler ${ }^{4}$ & \\
\hline & C. ferdinandiana (Barb.Rodr.) Szlach. et al. ${ }^{4}$ & \\
\hline & C. neuwiedii (Rchb.f.) S.Koehler ${ }^{4}$ & \\
\hline & C. pumila (Hook.) Szlach. et al. ${ }^{4}$ & \\
\hline & Cirrhaea dependens (Lodd.) Loudon ${ }^{4}$ & \\
\hline & Coppensia flexuosa (Sims) Campacci & JAL 7300 \\
\hline & C. hookeri (Rolfe) F.Barros \& L.Guimarães ${ }^{4}$ & \\
\hline & C. montana (Barb.Rodr.) Campacci ${ }^{4}$ & \\
\hline & C. varicosa (Lindl.) Campacci ${ }^{4}$ & \\
\hline & Corymborchis flava (Sw.) Kuntze & JAL 7260 \\
\hline & Cyclopogon calophyllus Barb.Rodr. ${ }^{4}$ & \\
\hline & C. congestus (Vell.) Hoehne & JAL 6834 \\
\hline & C. elatus (Sw.) Schltr. & JAL 7481 \\
\hline & C. variegatus Barb.Rodr. ${ }^{4}$ & \\
\hline & Dryadella aviceps (Rchb.f.) Luer ${ }^{4}$ & \\
\hline & Encyclia patens Hook. $^{4}$ & \\
\hline & Epidendrum armeniacum Lindl. ${ }^{4}$ & \\
\hline & E. chlorinum Barb.Rodr. ${ }^{4}$ & \\
\hline & E. difforme Jacq. ${ }^{4}$ & \\
\hline & E. henschenii Barb.Rodr. ${ }^{4}$ & \\
\hline & E. latilabre Lindl. ${ }^{4}$ & \\
\hline & E. martianum Lindl. $^{4}$ & \\
\hline & E. ochrochlorum Barb.Rodr. ${ }^{4}$ & \\
\hline & E. paniculatum Ruiz \& Pav. ${ }^{4}$ & \\
\hline & E. proligerum Barb.Rodr. ${ }^{4}$ & \\
\hline & E. secundum Jacq. & JAL 6463 \\
\hline & Eulophia alta (L.) Fawc. \& Rendle ${ }^{4}$ & \\
\hline
\end{tabular}




\begin{tabular}{|c|c|c|}
\hline Família & Espécie & Voucher \\
\hline & Eurystyles actinosophila (Barb.Rodr.) Schltr. & JAL 7427 \\
\hline & Galeandra beyrichii Rchb.f. & JAL 7367 \\
\hline & Gomesa crispa (Lindl.) Klotzsch \& Rchb.f. ${ }^{4}$ & \\
\hline & G. gomezoides (Barb.Rodr.) Pabst ${ }^{4}$ & \\
\hline & G. handroi (Hoehne) Pabst ${ }^{4}$ & \\
\hline & G. recurva $\mathrm{R} . \mathrm{Br}^{4}$ & \\
\hline & Govenia utriculata (Sw.) Lindl. & JAL 7306 \\
\hline & Grandiphyllum auricula (Vell.) Docha Neto ${ }^{4}$ & \\
\hline & G. hians (Lindl.) Docha Neto ${ }^{4}$ & \\
\hline & Grobya amherstiae Lindl. $^{4}$ & \\
\hline & Habenaria araneiflora Barb.Rodr. ${ }^{4}$ & \\
\hline & H. glaucophylla Barb.Rodr. ${ }^{4}$ & \\
\hline & H. johannensis Barb.Rodr. ${ }^{4}$ & \\
\hline & H. josephensis Barb.Rodr. & JAL 6635 \\
\hline & H. parviflora Lindl. & JAL 7334 \\
\hline & H. paulistana J.A.N.Batista \& Bianchetti ${ }^{4}$ & \\
\hline & H. pleiophylla Hoehne \& Schltr. ${ }^{4}$ & \\
\hline & H. warmingii Rchb.f. \& Warm. ${ }^{4}$ & \\
\hline & Habenaria sp. 1 & JAL 7301 \\
\hline & Hapalorchis lineatus (Lindl.) Schltr. ${ }^{4}$ & \\
\hline & H. micranthus (Barb.Rodr.) Hoehne ${ }^{4}$ & \\
\hline & Heterotaxis brasiliensis (Brieger \& Illg) F.Barros ${ }^{4}$ & \\
\hline & Ionopsis utricularioides (Sw.) $\mathrm{Lindl}^{4}$ & \\
\hline & Isabelia violacea (Lindl.) van den Berg \& M.W.Chase $\mathrm{e}^{4}$ & \\
\hline & I. virginalis Barb.Rodr. ${ }^{4}$ & \\
\hline & Isochilus linearis (Jacq.) R.Br. ${ }^{4}$ & \\
\hline & Liparis nervosa (Thumb.) Lindl. & JAL 7345 \\
\hline & Lockhartia lunifera (Lindl.) Rchb.f. ${ }^{4}$ & \\
\hline & Lophiaris pumila (Lindl.) Braem ${ }^{4}$ & \\
\hline & Malaxis excavata (Lindl.) Kuntze ${ }^{4}$ & \\
\hline & M. leucaimata Barb. Rodr. ${ }^{4}$ & \\
\hline & Mesadenella atroviridis (Barb.Rodr.) Garay ${ }^{4}$ & \\
\hline & Maxillaria cuspidata (Lindl.) Garay & JAL 7440 \\
\hline & Miltonia regnellii Rchb.f. $^{4}$ & \\
\hline & Notylia nemorosa Barb.Rodr. ${ }^{4}$ & \\
\hline & Octomeria crassifolia Lindl. $^{4}$ & \\
\hline & O. diaphana Lindl. ${ }^{4}$ & \\
\hline & Oeceoclades maculata (Lindl.) Lindl. ${ }^{4}$ & \\
\hline & Pelexia oestrifera (Rchb.f. \& Warm.) Schltr. ${ }^{4}$ & \\
\hline & Pleurothallis schenkii Luer ${ }^{4}$ & \\
\hline & Polystachya caespitosa Barb.Rodr. ${ }^{4}$ & \\
\hline & P. estrellensis Rchb.f. ${ }^{4}$ & \\
\hline & P. montana Barb.Rodr. ${ }^{4}$ & \\
\hline
\end{tabular}




\begin{tabular}{|c|c|c|}
\hline Família & Espécie & Voucher \\
\hline & P. oligantha (Sw.) Lindl. ${ }^{4}$ & \\
\hline & P. stachyodes (Sw.) Lindl. ${ }^{4}$ & \\
\hline & Promenaea rollissonii (Lindl.) Lindl. ${ }^{4}$ & \\
\hline & Prosthechea bulbosa (Vell.) W.E.Higgins ${ }^{4}$ & \\
\hline & P. calamaria (Lindl.) W.E.Higgins ${ }^{4}$ & \\
\hline & Prosthechea sp. 1 & JAL 6985 \\
\hline & Psilochilus modestus Barb. Rodr. ${ }^{4}$ & \\
\hline & Pteroglossa glazioviana (Cogn.) Garay ${ }^{4}$ & \\
\hline & Rhetinantha notylioglossa (Rchb.f.) M.A.Blanco ${ }^{4}$ & \\
\hline & Rodriguezia decora (Lem.) Rchb.f. & JAL 7419 \\
\hline & Sacoila lanceolata (Aubl.) Garay & JAL 6910 \\
\hline & Sarcoglottis fasciculata (Vell.) Schltr. ${ }^{4}$ & \\
\hline & Sauroglossum nitidum (Vell.) Schltr. ${ }^{4}$ & \\
\hline & Specklinia grobyi (Batem. ex Lindl.) F.Barros ${ }^{4}$ & \\
\hline & S. uniflora (Lindl.) Pridgeon \& M.W.Chase ${ }^{4}$ & \\
\hline & Stanhopea lietzei (Regel) Schltr. ${ }^{4}$ & \\
\hline & Stelis ephemera (Lindl.) Pridgeon \& M.W.Chase ${ }^{4}$ & \\
\hline & S. hypnicola (Lindl.) Pridgeon \& M.W.Chase ${ }^{4}$ & \\
\hline & Stigmatosema polyaden (Vell.) Garay ${ }^{4}$ & \\
\hline & Vanilla bahiana Hoehne ${ }^{4}$ & \\
\hline & V. edwallii Hoehne ${ }^{4}$ & \\
\hline & Wullschlaegelia aphylla (Sw.) Rchb.f. & JAL 7323 \\
\hline & Zygopetalum maculatum (Kunth) Garay ${ }^{4}$ & \\
\hline & Zygostates lunata Lindl. ${ }^{4}$ & \\
\hline & Indet. 1 & JAL 6704 \\
\hline & Indet. 2 & JAL 7261 \\
\hline \multirow[t]{2}{*}{ Oxalidaceae } & Oxalis rhombeo-ovata A.St.-Hil. & JAL 6832 \\
\hline & O. triangularis A.St.-Hil. ${ }^{\S}$ & JAL 6897 \\
\hline \multirow[t]{6}{*}{ Passifloraceae } & Passiflora amethystina J.C.Mikan & JAL 6763 \\
\hline & P. capsularis $\mathrm{L}$. & JAL 7311 \\
\hline & P. edulis $\mathrm{Sims}^{\S}$ & JAL 7276 \\
\hline & P. organensis Gardner & JAL 7322 \\
\hline & P. sidifolia M.Roem. & JAL 6764 \\
\hline & P. suberosa L. & JAL 7013 \\
\hline Pentaphyllaceae & Ternstroemia brasiliensis Cambess. ${ }^{1}$ & \\
\hline Peraceae & Pera obovata Baill. & JAL 6604 \\
\hline \multirow[t]{2}{*}{ Phyllanthaceae } & Phyllanthus tenellus Roxb. ${ }^{\S}$ & JAL 7248 \\
\hline & Savia dictyocarpa Müll.Arg. ${ }^{2}$ & \\
\hline \multirow[t]{2}{*}{ Phytolaccaceae } & Gallesia integrifolia (Spreng.) Harms ${ }^{2}$ & \\
\hline & Seguieria langsdorffii Moq. ${ }^{2}$ & \\
\hline \multirow{2}{*}{$\begin{array}{l}\text { Picramniaceae (J.R. Pirani } \\
\text { - SPF) }\end{array}$} & Picramnia parvifolia Engl. & JAL 7489 \\
\hline & Picramnia ramiflora Planch. ${ }^{3}$ & \\
\hline Pinaceae & Pinus sp. $1^{\S}$ & JAL 6922 \\
\hline
\end{tabular}




\begin{tabular}{|c|c|c|}
\hline Família & Espécie & Voucher \\
\hline \multirow{18}{*}{$\begin{array}{l}\text { Piperaceae (E.F. Guimarães } \\
\& \text { M. Carvalho-Silva - RB) }\end{array}$} & Manekia obtusa (Miq.) Arias et al. & JAL 7044 \\
\hline & Peperomia alata Ruiz \& Pav. & JAL 7296 \\
\hline & P. blanda (Jacq.) Kunth & JAL 7384 \\
\hline & P. nitida Dahlst. & JAL 7041 \\
\hline & P. rotundifolia $($ L.) Kunth & JAL 8650 \\
\hline & P. urocarpa Fisch. \& C.A.Mey. & JAL 7422 \\
\hline & Piper aduncum $\mathrm{L}$. & JAL 6730 \\
\hline & P. amalago L. ${ }^{3}$ & \\
\hline & P. corcovadensis (Miq.) C.DC. & JAL 7282 \\
\hline & P. crassinervium Kunth & JAL 6731 \\
\hline & P. gaudichaudianum Kunth & JAL 6600 \\
\hline & P. glabratum Kunth & JAL 6749 \\
\hline & P. hayneanum C.DC. & JAL 7431 \\
\hline & P. richardiifolium Kunth & JAL 7034 \\
\hline & Piper sp. 1 & JAL 6710 \\
\hline & Piper sp. 2 & JAL 6911 \\
\hline & Piper sp. 3 & JAL 7046 \\
\hline & Piper sp. 4 & JAL 7065 \\
\hline \multirow[t]{2}{*}{ Plantaginaceae } & Plantago major L. ${ }^{\S}$ & JAL 6796 \\
\hline & Scoparia dulcis L. ${ }^{\S}$ & JAL 7082 \\
\hline \multirow{20}{*}{$\begin{array}{l}\text { Poaceae (P.L. Viana - } \\
\text { BHCB) }\end{array}$} & Andropogon bicornis $\mathrm{L}$. & JAL 7244 \\
\hline & Bambusa tuldoides Munro" & JAL 6972 \\
\hline & Chusquea cf. bambusoides (Raddi) Hack. & JAL 7458 \\
\hline & C. cf. leptophylla Nees & JAL 6716 \\
\hline & Dichanthelium hebotes (Trin.) Zuloaga & JAL 6833 \\
\hline & D. sabulorum (Lam.) Gould \& C.A.Clark & JAL 6616 \\
\hline & Eragrostis rufescens Schrad. ex Schult. & JAL 7388 \\
\hline & Ichnanthus pallens (Sw.) Munro ex Benth. & JAL 7366 \\
\hline & Lasiacis cf. sorghoidea (Desv. ex Ham.) Hitchc. \& Chase & JAL 7467 \\
\hline & Melinis minutiflora P.Beauv. $\S$ & JAL 6665 \\
\hline & M. repens (Willd.) Zizka & JAL 7394 \\
\hline & Merostachis sp. 1 & JAL 7459 \\
\hline & Olyra humilis Nees & JAL 7073 \\
\hline & Panicum sp. 1 & JAL 7033 \\
\hline & Parodiolyra micrantha (Kunth) Davidse \& Zuloaga & JAL 6777 \\
\hline & Paspalum corcovadense Raddi ${ }^{\S}$ & JAL 6617 \\
\hline & P. urvillei Steud $\S$ & JAL 7001 \\
\hline & Pennisetum purpureum Schumach. ${ }^{\S}$ & JAL 6615 \\
\hline & Pharus lappulaceus Aubl. & JAL 7308 \\
\hline & Phyllostachys aurea Rivière \& C.Rivière ${ }^{\#}$ & JAL 6826 \\
\hline
\end{tabular}




\begin{tabular}{|c|c|c|}
\hline Família & Espécie & Voucher \\
\hline & Polypogon elongatus Kunth & JAL 7020 \\
\hline & Pseudechinolaena polystachya (Kunth) Stapf & JAL 6672 \\
\hline & Setaria sulcata Raddi $^{\S}$ & JAL 7280 \\
\hline & Sporobolus indicus (L.) R.Br. & JAL 7022 \\
\hline & Urochloa brizantha (Hochst. ex A.Rich.) R.D.Webster $\$$ & JAL 6935 \\
\hline \multirow[t]{4}{*}{ Polygalaceae } & Bredemeyera kunthiana (A.St.-Hil) Klotzsch ex A.W.Benn. & JAL 7455 \\
\hline & Diclidanthera laurifolia Mart. & JAL 6995 \\
\hline & Polygala monninoides Kunth & JAL 6625 \\
\hline & P. paniculata $\mathrm{L}$. & JAL 6791 \\
\hline Polygonaceae & Polygonum capitatum Buch.-Ham. ex D.Don ${ }^{\S}$ & JAL 7473 \\
\hline \multirow{11}{*}{$\begin{array}{l}\text { Polypodiaceae (T.E. } \\
\text { Almeida, A. Salino - BHBC) }\end{array}$} & Campyloneurum nitidum (Kaulf.) C.Presl & JAL 6662 \\
\hline & C. rigidum J.Sm. & JAL 8660 \\
\hline & Microgramma squamulosa (Kaulf.) de la Sota & JAL 6620 \\
\hline & M. tecta (Kaulf.) Alston & JAL 8645 \\
\hline & Niphidium crassifolium (L.) Lellinger & JAL 7454 \\
\hline & Pecluma recurvata (Kaulf.) M.G.Price & JAL 6670 \\
\hline & Phlebodium pseudoaureum (Cav.) Lellinger & JAL 7397 \\
\hline & Pleopeltis pleopeltifolia (Raddi) Alston & JAL 6723 \\
\hline & Polypodium sp. 1 & JAL 6659 \\
\hline & Serpocaulon catharinae (Langsd. \& Fisch.) A.R.Sm. & JAL 7373 \\
\hline & S. latipes (Langsd. \& Fisch.) A.R.Sm. & JAL 7396 \\
\hline Portulacaceae & Talinum paniculatum (Jacq.) Gaertn. & JAL 6988 \\
\hline Proteaceae & Roupala montana var. brasiliensis (Sleumer) K.S. Edwards & JAL 6645 \\
\hline \multirow{11}{*}{$\begin{array}{l}\text { Pteridaceae (A. Salino - } \\
\text { BHCB, J.Prado - SP) }\end{array}$} & Adiantopsis radiata (L.) Fée & JAL 6875 \\
\hline & Adiantum lorentzii Hieron. & JAL 7357 \\
\hline & A. pentadactylon Langsd. \& Fisch. & JAL 7362 \\
\hline & A. raddianum C.Presl & JAL 6678 \\
\hline & A. subcordatum $\mathrm{Sw}$. & JAL 6782 \\
\hline & Doryopteris patula Fée & JAL 6820 \\
\hline & Pteris denticulata $\mathrm{Sw}$. & JAL 7049 \\
\hline & P. lechleri Mett. & JAL 7398 \\
\hline & P. splendens Kaulf. & JAL 7281 \\
\hline & P. vittata $\mathrm{L}$. & JAL 7409 \\
\hline & Vittaria lineata $(\mathrm{L}.) \mathrm{Sm}$. & JAL 7448 \\
\hline Ranunculaceae & Clematis dioica $\mathrm{L}$. & JAL 7332 \\
\hline \multirow[t]{3}{*}{ Rhamnaceae } & Colubrina glandulosa Perkins ${ }^{2,3}$ & \\
\hline & Gouania virgata Reissek & JAL 7246 \\
\hline & Rhamnidium elaeocarpum Reissek ${ }^{2}$ & \\
\hline \multirow[t]{2}{*}{ Rosaceae } & Eriobotrya japonica (Thunb.) Lindl. ${ }^{\S}$ & JAL 6643 \\
\hline & Prunus myrtifolia (L.) Urb. & JAL 6708 \\
\hline
\end{tabular}




\begin{tabular}{|c|c|c|}
\hline Família & Espécie & Voucher \\
\hline & Rubus brasiliensis Mart. & JAL 7417 \\
\hline & R. rosifolius $\mathrm{Sm}$. & JAL 6753 \\
\hline & R. sellowii Cham. \& Schltdl. & JAL 6754 \\
\hline \multirow[t]{38}{*}{ Rubiaceae } & Alseis floribunda Schott $^{2}$ & \\
\hline & Amaioua guianensis Aubl. ${ }^{2}$ & \\
\hline & Bathysa australis (A.St.-Hil.) K.Schum. & JAL 6664 \\
\hline & Borreria latifolia (Aubl.) K.Schum. ${ }^{\S}$ & JAL 7305 \\
\hline & B. verticillata (L.) G.Mey. ${ }^{\S}$ & JAL 6594 \\
\hline & Chiococca alba (L.) Hitch. & JAL 7404 \\
\hline & Coccocypselum geophiloides Wawra & JAL 7032 \\
\hline & C. lanceolatum (Ruiz \& Pav.) Pers. & JAL 6992 \\
\hline & Coffea arabica $\mathrm{L} . \S$ & JAL 7433 \\
\hline & Cordiera concolor (Cham.) Kuntze & JAL 7245 \\
\hline & Coussarea contracta (Walp.) Müll.Arg., ${ }^{2,3}$ & \\
\hline & C. nodosa (Benth.) Müll.Arg. ${ }^{2}$ & \\
\hline & Coutarea hexandra (Jacq.) K.Schum. & JAL 7497 \\
\hline & Diodella apiculata (Willd. ex Roem. \& Schult.) Delprete & JAL 6622 \\
\hline & Emmeorhiza umbellata (Spreng.) K.Schum. & JAL 6700 \\
\hline & Galianthe laxa (Cham. \& Schltdl.) E.L.Cabral & JAL 7259 \\
\hline & Galium hypocarpium (L.) Endl. ex Griseb. & JAL 6819 \\
\hline & Guettarda viburnoides Cham. \& Schltdl. ${ }^{2}$ & \\
\hline & Ixora brevifolia Benth. & JAL 6937 \\
\hline & I. gardneriana Benth. & JAL 7406 \\
\hline & I. venulosa Benth. $^{2}$ & \\
\hline & Manettia cordifolia Mart. & JAL 6461 \\
\hline & M. gracilis Cham. \& Schltdl. & JAL 6627 \\
\hline & M. luteo-rubra (Vell.) Benth. & JAL 6629 \\
\hline & Palicourea marcgravii A.St.-Hil. & JAL 6607 \\
\hline & Posoqueria acutifolia Mart. & JAL 7517 \\
\hline & P. latifolia (Rudge) Schult. ${ }^{1,2}$ & \\
\hline & Psychotria carthagenensis Jacq. & JAL 7057 \\
\hline & P. forsteronioides Müll.Arg. & JAL 6603 \\
\hline & P. hastisepala Müll.Arg. & JAL 7368 \\
\hline & P. hoffmannseggiana (Willd. ex Schult.) Müll.Arg. & JAL 6602 \\
\hline & P. nuda (Cham. \& Schltdl.) Wawra ${ }^{2}$ & \\
\hline & P. ruelliifolia (Cham. \& Schltdl.) Müll.Arg. & JAL 6941 \\
\hline & P. suterella Müll.Arg. & JAL 6640 \\
\hline & P. vellosiana Benth. & JAL 6474 \\
\hline & Randia armata (Sw.) DC. ${ }^{2}$ & \\
\hline & Richardia brasiliensis Gomes ${ }^{\S}$ & JAL 7390 \\
\hline & Rudgea gardenioides (Cham.) Müll.Arg., ${ }^{1,3}$ & \\
\hline
\end{tabular}




\begin{tabular}{|c|c|c|}
\hline Família & Espécie & Voucher \\
\hline & R. jasminoides (Cham.) Müll.Arg. ${ }^{1}$ & \\
\hline & R. minor (Cham.) Standl. subsp. minor ${ }^{2,3}$ & \\
\hline & Indet. 1 & JAL 6679 \\
\hline \multirow[t]{14}{*}{ Rutaceae } & Balfourodendron riedelianum (Engl.) Engl. ${ }^{2}$ & \\
\hline & Citrus latifolia (Tanaka ex Yu.Tanaka) Tanaka ${ }^{\#}$ & JAL 6847 \\
\hline & C. $\operatorname{limon}(\mathrm{L}$.$) Burm.f. { }^{\S}$ & JAL 7472 \\
\hline & Dictyoloma vandellianum A.Juss. ${ }^{2}$ & \\
\hline & Esenbeckia febrifuga (A.St.-hil.) A.Juss. ex Mart. ${ }^{2,3}$ & \\
\hline & E. grandiflora Mart. & JAL 8648 \\
\hline & E. leiocarpa Engl. $^{2}$ & \\
\hline & Galipea jasminiflora (A.St.-Hil.) Engl. ${ }^{2}$ & \\
\hline & Metrodorea nigra A.St.-Hil. ${ }^{2}$ & \\
\hline & M. stipularis Mart. $^{2}$ & \\
\hline & Zanthoxylum caribaeum Lam. $^{2}$ & \\
\hline & Z. fagara (L.) Sarg. & \\
\hline & Z. monogynum A.St.-Hil. ${ }^{2}$ & \\
\hline & Z. rhoifolium Lam. ${ }^{2}$ & \\
\hline Sabiaceae & Meliosma sellowii Urb. & JAL 6468 \\
\hline \multirow[t]{7}{*}{ Salicaceae } & Casearia decandra Jacq. ${ }^{1,2,3}$ & \\
\hline & C. gossypiosperma Briq. $^{2}$ & \\
\hline & C. obliqua Spreng. ${ }^{1,3}$ & \\
\hline & C. sylvestris $\mathrm{Sw}$. & JAL 6921 \\
\hline & Prockia crucis P.Browne ex L. & JAL 7007 \\
\hline & Indet. 1 & JAL 7492 \\
\hline & Xylosma ciliatifolia (Clos) Eichler & JAL 6701 \\
\hline Santalaceae & Phoradendron crassifolium (Pohl ex DC.) Eichler & JAL 6734 \\
\hline \multirow{15}{*}{$\begin{array}{l}\text { Sapindaceae (M.S. Ferrucci } \\
\text { - CTES) }\end{array}$} & Allophylus edulis (A.St.-Hil. et al.) Hieron. ex Niedrl. & JAL 6855 \\
\hline & A. petiolulatus Radlk. ${ }^{2}$ & \\
\hline & Cupania vernalis Cambess. ${ }^{1,2,3}$ & \\
\hline & C. zanthoxyloides Radlk. & JAL 6839 \\
\hline & Dodonaea viscosa Jacq. & JAL 6597 \\
\hline & Matayba guianensis Aubl. ${ }^{2}$ & \\
\hline & M. juglandifolia (Cambess.) Radlk. ${ }^{2,3}$ & \\
\hline & Matayba sp. 1 & JAL 7491 \\
\hline & Paullinia rhomboidea Radlk. & JAL 6732 \\
\hline & Serjania communis Cambess. & JAL 6785 \\
\hline & S. laruotteana Cambess. & JAL 6596 \\
\hline & S. multiflora Cambess. & JAL 7466 \\
\hline & S. paradoxa Radlk. & JAL 7407 \\
\hline & S. reticulata Cambess. & JAL 7352 \\
\hline & Urvillea ulmacea Kunth & JAL 6712 \\
\hline
\end{tabular}




\begin{tabular}{|c|c|c|}
\hline Família & Espécie & Voucher \\
\hline & Indet. 1 & JAL 7514 \\
\hline \multirow[t]{3}{*}{ Sapotaceae } & Chrysophyllum gonocarpum (Mart. \& Eichler ex Miq.) Engl., 3 & \\
\hline & C. marginatum (Hook. \& Arn.) Radlk. ${ }^{1,2}$ & \\
\hline & Pouteria caimito (Ruiz \& Pav.) Radlk. ${ }^{2}$ & \\
\hline Schoepfiaceae & Schoepfia brasiliensis A.DC. ${ }^{2}$ & \\
\hline Scrophulariaceae & Buddleja stachyoides Cham. \& Schltdl. & JAL 6661 \\
\hline \multirow[t]{3}{*}{ Smilacaceae } & Smilax elastica Griseb. & JAL 6642 \\
\hline & Smilax sp. 1 & JAL 7383 \\
\hline & Smilax sp. 2 & JAL 7432 \\
\hline \multirow{30}{*}{$\begin{array}{l}\text { Solanaceae (L.L. Giacomin, } \\
\text { J.R. Stehmann - BHCB) }\end{array}$} & Athenaea picta (Mart.) Sendtn. & JAL 7488 \\
\hline & Aureliana fasciculata (Vell.) Sendtn. & JAL 6460 \\
\hline & Brugmansia suaveolens (Willd.) Bercht. \& J.Pres $1^{\S}$ & JAL 6863 \\
\hline & Brunfelsia brasiliensis (Spreng.) L.B.Sm. \& Downs & JAL 6852 \\
\hline & Capsicum baccatum L. ${ }^{\S}$ & JAL 7298 \\
\hline & Capsicum sp. 1 & JAL 7000 \\
\hline & Cestrum axillare Vell. ${ }^{1}$ & \\
\hline & C. schlechtendalii G.Don & JAL 6761 \\
\hline & C. strigilatum Ruiz \& Pav. & JAL 6729 \\
\hline & Nicotiana tabacum $\mathrm{L} . \S$ & JAL 6958 \\
\hline & Sessea brasiliensis Toledo & JAL 6696 \\
\hline & Solanum americanum Mill. & JAL $6789 \S$ \\
\hline & S. argenteum Dunal $^{2,3}$ & \\
\hline & S. betaceum Cav. ${ }^{2}$ & \\
\hline & S. bullatum Vell. ${ }^{1}$ & \\
\hline & S. campaniforme Roem. \& Schult. & JAL 6888 \\
\hline & S. cernuит Vell. ${ }^{2}$ & \\
\hline & S. concinuum Sendtn. & JAL 6752 \\
\hline & S. decompositiflorum Schott ex Sendtn. & JAL 7016 \\
\hline & S. didymum Dunal & JAL 6626 \\
\hline & S. granulosoleprosum Dunal & JAL 6694 \\
\hline & S. inodorum Vell. & JAL 7426 \\
\hline & S. palinacanthum Dunal ${ }^{\S}$ & JAL 7056 \\
\hline & S. paniculatum L. ${ }^{\S}$ & JAL 7438 \\
\hline & S. pseudoquina A.St.-Hil. & JAL 7377 \\
\hline & S. rufescens Sendtn. & JAL 6786 \\
\hline & S. sanctae-catharinae Dunal & JAL 7292 \\
\hline & S. sciadostylis (Sendtn.) Bohs & JAL 6982 \\
\hline & S. swartzianum Roem. \& Schult. ${ }^{2}$ & \\
\hline & S. variabile Mart. & LBS 73 \\
\hline Styracaceae & Styrax latifolius Pohl ${ }^{2,3}$ & \\
\hline
\end{tabular}




\begin{tabular}{|c|c|c|}
\hline Família & Espécie & Voucher \\
\hline & S. pohlii A.DC. & JAL 6962 \\
\hline \multirow{5}{*}{$\begin{array}{l}\text { Symplocaceae (J.L.M. } \\
\text { Aranha Filho - UEC) }\end{array}$} & Symplocos celastrinea Mart. & JAL 7490 \\
\hline & S. glandulosomarginata Hoehne ${ }^{1}$ & \\
\hline & S. pubescens Klotzsch ex Benth. ${ }^{1}$ & \\
\hline & S. revoluta Casar. & JAL 6697 \\
\hline & S. tetrandra Mart. & JAL 7391 \\
\hline Theaceae & Laplacea fructicosa (Schrad.) Kobuski ${ }^{1,2,3}$ & \\
\hline \multirow{5}{*}{$\begin{array}{l}\text { Thelypteridaceae (T.E. } \\
\text { Almeida, A. Salino - BHCB) }\end{array}$} & Macrothelypteris torresiana (Gaudich.) Ching ${ }^{\S}$ & JAL 8641 \\
\hline & Thelypteris amambayensis Ponce & JAL 8658 \\
\hline & T. dentata (Forssk.) E.P.St.John ${ }^{\S}$ & JAL 7408 \\
\hline & T. hispidula (Decne.) C.F. Reed & JAL 7408 \\
\hline & T. cf. lugubris (Mett.) R.M.Tryon \& A.F.Tryon & \\
\hline Thymelaeaceae & Daphnopsis fasciculata (Meisn.) Nevling & JAL 6780 \\
\hline Tropaeolaceae & Tropaeolum majus L. ${ }^{\S}$ & JAL 6959 \\
\hline Turneraceae & Turnera capitata Cambess. & JAL 6906 \\
\hline Typhaceae & Typha latifolia $\mathrm{L}$. & JAL 7518 \\
\hline \multirow[t]{6}{*}{ Urticaceae } & Boehmeria caudata $\mathrm{Sw}$. & JAL 6987 \\
\hline & Cecropia glaziovi Snethl. & JAL 7453 \\
\hline & C. hololeuca Miq. $^{2}$ & \\
\hline & C. pachystachya Trécul & JAL 6868 \\
\hline & Pilea pubescens Liebm. & JAL 7319 \\
\hline & Urera baccifera (L.) Gaudich. ex Wedd. & JAL 7058 \\
\hline Valerianaceae & Valeriana scandens $\mathrm{L}$. & JAL 6467 \\
\hline \multirow[t]{8}{*}{ Verbenaceae } & Citharexylum myrianthum Cham. ${ }^{2}$ & \\
\hline & Duranta erecta L." & JAL 7070 \\
\hline & Lantana fucata Lindl. & JAL 6471 \\
\hline & Lippia brasiliensis (Link) T.R.S.Silva & JAL 7303 \\
\hline & L. lippioides (Cham.) Rusby & JAL 7252 \\
\hline & Stachytarpheta cayennensis (Rich.) Vahl ${ }^{\S}$ & JAL 6963 \\
\hline & Verbena litoralis $\mathrm{Kunth}^{\S}$ & JAL 6956 \\
\hline & V. rigida Spreng. ${ }^{\S}$ & JAL 6871 \\
\hline \multirow[t]{2}{*}{ Violaceae } & Anchietea pyrifolia (Mart.) G.Don & JAL 6798 \\
\hline & Hybanthus atropurpureus (A.St.-Hil.) Taub. & JAL 6745 \\
\hline \multirow[t]{4}{*}{ Vitaceae } & Cissus serroniana (Glaz.) Lombardi & JAL 7295 \\
\hline & C. striata subsp. argentina (Suess.) Lombardi & JAL 6469 \\
\hline & C. sulcicaulis (Baker) Planch. & JAL 6756 \\
\hline & C. verticillata (L.) Nicolson \& C.E.Jarvis subsp. verticillata & JAL 6727 \\
\hline \multirow[t]{2}{*}{ Vochysiaceae } & Callisthene minor Mart. & LBS 71 \\
\hline & Qualea dichotoma (Mart.) Warm. ${ }^{2,3}$ & \\
\hline
\end{tabular}




\begin{tabular}{llc}
\hline Família & Espécie & Voucher \\
\hline & $\begin{array}{l}\text { Q. } \text { multiflora } \text { subsp. pubescens (Mart.) Stafleu } \\
\text { Vochysia magnifica } \text { Warm., }\end{array}$ & JAL 7434 \\
& V. tucanorum Mart. & JAL 6711 \\
Woodsiaceae (A. Salino - & Diplazium plantaginifolium (L.) Urb. & JAL 7271 \\
BHCB) & Kniphofia uvaria (L.) Oken ${ }^{\#}$ & JAL 7436 \\
Xanthorrhoeaceae & Xyris laxifolia Mart. & JAL 7336 \\
Xyridaceae & Hedychium coronarium J.Koenig $\$$ & JAL 7327 \\
Zingiberaceae & &
\end{tabular}

